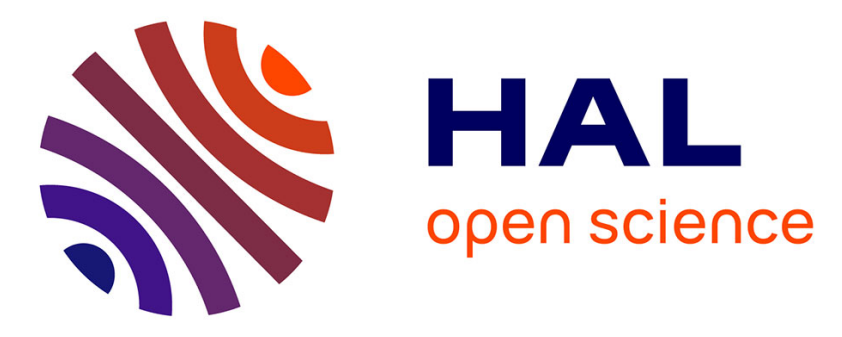

\title{
Statistical properties of single-mode fiber coupling of satellite-to-ground laser links partially corrected by adaptive optics
}

Lucien Canuet, Nicolas Vedrenne, Jean-Marc Conan, Cyril Petit, Géraldine Artaud, Angélique Rissons, Jérôme Lacan

\section{To cite this version:}

Lucien Canuet, Nicolas Vedrenne, Jean-Marc Conan, Cyril Petit, Géraldine Artaud, et al.. Statistical properties of single-mode fiber coupling of satellite-to-ground laser links partially corrected by adaptive optics. Journal of the Optical Society of America. A Optics, Image Science, and Vision, 2018, 1 (35), pp.148-162. 10.1364/JOSAA.35.000148 . hal-01904583

\section{HAL Id: hal-01904583 \\ https://hal.science/hal-01904583}

Submitted on 25 Oct 2018

HAL is a multi-disciplinary open access archive for the deposit and dissemination of scientific research documents, whether they are published or not. The documents may come from teaching and research institutions in France or abroad, or from public or private research centers.
L'archive ouverte pluridisciplinaire HAL, est destinée au dépôt et à la diffusion de documents scientifiques de niveau recherche, publiés ou non, émanant des établissements d'enseignement et de recherche français ou étrangers, des laboratoires publics ou privés. 


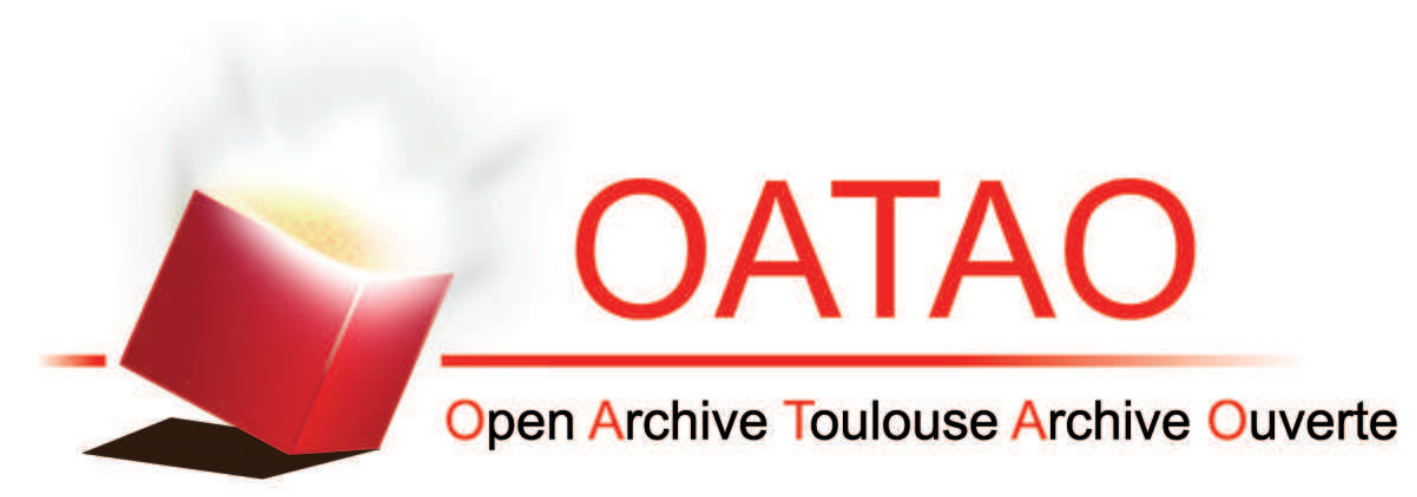

\section{Open Archive Toulouse Archive Ouverte (OATAO)}

OATAO is an open access repository that collects the work of some Toulouse researchers and makes it freely available over the web where possible.

This is an author's version published in: https://oatao.univ-toulouse.fr/20968

Official URL:http://doi.org/10.1364/JOSAA.35.000148

\section{To cite this version :}

Canuet, Lucien and Vedrenne, Nicolas and Conan, Jean-Marc and Petit, Cyril and Artaud, Géraldine and Rissons, Angélique and Lacan, Jérôme Statistical properties of single-mode fiber coupling of satellite-to-ground laser links partially corrected by adaptive optics. (2018) Journal of the Optical Society of America A, 1 (35). 148-162. ISSN 1084-7529

Any correspondence concerning this service should be sent to the repository administrator: tech-oatao@listes-diff.inp-toulouse.fr 


\title{
Statistical properties of single-mode fiber coupling of satellite-to-ground laser links partially corrected by adaptive optics
}

\author{
Lucien Canuet, ${ }^{1,2,3, *}$ Nicolas Védrenne, ${ }^{2}$ Jean-Marc Conan, ${ }^{2}$ Cyril Petit, ${ }^{2}$ Geraldine Artaud, ${ }^{1}$ \\ Angelique Rissons, ${ }^{3}$ and Jerome Lacan ${ }^{3}$ \\ ${ }^{1}$ CNES, Centre National d'Études Spatiales, 18, av. Edouard Belin, 31401 Toulouse Cedex 9, France \\ ${ }^{2}$ ONERA, The French Aerospace Lab, Theoretical and Applied Optics Department, 92322 Châtillon Cedex, France \\ ${ }^{3}$ University of Toulouse; ISAE-Supaéro; TeSA, Toulouse, France \\ ${ }^{*}$ Corresponding author: lucien.canuet@onera.fr
}

\begin{abstract}
In the framework of satellite-to-ground laser downlinks, an analytical model describing the variations of the instantaneous coupled flux into a single-mode fiber after correction of the incoming wavefront by partial adaptive optics (AO) is presented. Expressions for the probability density function and the cumulative distribution function as well as for the average fading duration and fading duration distribution of the corrected coupled flux are given. These results are of prime interest for the computation of metrics related to coded transmissions over correlated channels, and they are confronted by end-to-end wave-optics simulations in the case of a geosynchronous satellite (GEO)-to-ground and a low earth orbit satellite (LEO)-to-ground scenario. Eventually, the impact of different $\mathrm{AO}$ performances on the aforementioned fading duration distribution is analytically investigated for both scenarios.
\end{abstract}

OCIS codes: (010.1080) Active or adaptive optics; (010.3310) Laser beam transmission; (060.0060) Fiber optics and optical communications; (060.2605) Free-space optical communication.

https://doi.org/10.1364/JOSAA.35.000148

\section{INTRODUCTION}

While future satellite-to-ground laser communication systems promise extremely high data rates, reasonable costs of implementation in the short term will be attainable only by leveraging the benefits of existing single-mode components developed for fiber telecommunication. Yet, atmospheric turbulence severely degrades the injection efficiency of the incoming optical wave into such components, leading to signal fades and channel impairments. On one hand, the use of adaptive optics (AO) should contribute to reducing substantially the criticality of these fades by partially compensating turbulence-induced phase fluctuations. However, when very high injection stability is needed, complex and expensive $\mathrm{AO}$ systems have to be considered. On the other hand, the use of numerical mitigation techniques such as interleaving and forward error-correcting (FEC) codes, while limited by on-board resources, could scale down the specifications and cost of $\mathrm{AO}$ systems. In order to effectively and simultaneously assess the performances of $\mathrm{AO}$ and numerical techniques, quality of service metrics such as the coded packet (or symbol) error rates must be computed. The computation of such metrics will require the knowledge of the distribution of the fades' as well as interfades' durations of the instantaneous AO-corrected coupled flux into a singlemode fiber (SMF). The latter is subjected to variations conditioned by the turbulent wavefront distortions, themselves characterized by phase as well as log-amplitude fluctuations. Assuming stationary turbulence conditions, increasing the diameter of the receiver will tend to decrease the coupled flux fluctuations thanks to aperture averaging at the expense of a more demanding AO system. Under such circumstances, the influences of residual phase fluctuations and aperture-averaged scintillation have to be studied jointly. Furthermore, particularly in satellite-to-ground transmission scenarios, $\mathrm{AO}$ error budgets should not take the fitting error solely into account, as it may roughly overestimate the overall system performance. A more realistic error budgeting, representative of a partial $\mathrm{AO}$ correction, needs to be considered by including the fitting, aliasing, and the temporal errors of the control loop. This is especially important when considering transmission links from satellites with significant orbital velocities such as low earth orbit satellite (LEO)-to-ground downlinks. If not by experiments, the statistics of the aforementioned coupled flux fading after 
partial AO correction can usually be obtained by means of wave-optics numerical simulations that are nonetheless very time-consuming or too complex to implement when a good depiction of the involved physical phenomena is required.

Recently, an expression of the probability density function (PDF) of the instantaneous SMF coupling efficiency in the presence of atmospheric turbulence was suggested in [1]. Assuming that phase variations were dominant over log-amplitude fluctuations, the authors found that a Ricean distribution was a reasonable statistical approximation. This result can be found as well in [2], in which the effects of both wavefront distortion and amplitude scintillation are taken into account. The underlying hypothesis common to both of these contributions is that the distorted light propagating to the pupil is composed of statistically independent cells (or speckle) [3]. This may be a sufficiently good approximation when studying a perfect $\mathrm{AO}$ correction for which residual phase variance is, indeed, dominated by the fitting error. In the case of a partial $\mathrm{AO}$ correction, the lower-order residuals corresponding to low spatial frequencies are nonzero and are thus intrinsically highly correlating.

Determining the statistics of the turbulent instantaneous coupling efficiency is to some extent similar to determining those of the short-exposure Strehl ratio (SR). A simple analytical model that assumes perfect $\mathrm{AO}$ correction, and is based on the central limit theorem describing the probability distribution of the latter while taking into account log-amplitude and phase fluctuations can be found in [4]. The SR statistics give a rough estimation of the coupling efficiency statistics. The SR can, however, be shown to be a pessimistic estimator of the coupling efficiency [5]. The SR statistics are therefore not sufficient for the purpose of dimensioning data reliability mechanisms such as time interleavers and other coding techniques. Nevertheless, both quantities depend approximately on the variance of the residual wavefront [6], which can be directly expressed in terms of the coefficients of the modal decomposition of the wavefront. This approach was adopted in [7], where it was found that the residual wavefront variance is distributed as the sum of two independent $\chi^{2}$ random variables: the first characterizing the residual tip-tilt and the second higher-order modes. The final result of such an analysis presents similarities with the gamma distribution characterizing the temporal variability of the short-exposure SR derived in [8]. In both of these contributions, important statistical assumptions are made about the independence and/or identical distribution of residual modes or phase values measured by the wavefront sensor. Although these assumptions appear very appealing because of their convenience, they may not stem from a proper physical justification. Therefore, using similar modal decomposition [9] adapted to the problem at hand as well as widespread hypotheses regarding the wavefront statistics, the present paper emphasizes the derivation of analytical expressions of the coupled flux's probability density function (PDF), cumulative distribution function (CDF) and temporal characteristics [temporal autocovariance, average fading duration (AFD), average interfading duration (AIFD), fading duration distribution (FDD), and interfading duration distribution (IFDD)] and their validation by end-to-end wave-optics simulations.
The present contribution aims to remedy a shortfall in the literature regarding the modeling options of these statistics, which are needed for dimension data reliability mechanisms such as interleavers and/or error-correcting codes for future satellite-to-ground optical links. In this regard, the temporal characteristics of the propagation channel and the effect of $\mathrm{AO}$ correction on them have to be accurately modeled. Consequently, consideration should be given to the correlation of lower-order wavefront aberration residuals induced by the limitations of realistic $\mathrm{AO}$ systems. For instance, it is noted later in this paper that neglecting the latter can lead to relative variations of several tens of percent in terms of AFD and AIFD. Thus, the novelty of our contribution is to provide an analytical model that will allow one to infer the efficiency of such data reliability mechanisms accurately while accurately taking into account the impact of the temporal correlation of the coupled flux fluctuations, and the joint effect of fiber injection losses due to partial $\mathrm{AO}$ correction residuals and aperture-averaged scintillation. To the best of our knowledge, these features have not been taken into account in any previously published work, which either dealt with the statistics of the SR in the framework of imaging applications or did not consider the low-order $\mathrm{AO}$ residuals' (temporal and aliasing errors) impact on the temporal characteristics of the coupled flux. Therefore, in comparison to the models proposed in the aforementioned contributions, the model described in the present paper, although more complex, is more accurate and particularly relevant to satellite-to-ground optical communication applications.

After the introduction of some mathematical definitions and notations in Section 2, the coupling efficiency between the AO corrected wavefront and the SMF is given in Subsection 3.A. In Subsection 3.B, the addition of the log-amplitude fluctuations, so as to take into account the influence of scintillation on the coupled flux into the SMF, is presented. Statistical results including the PDF, CDF of the coupled flux, as well as the AFD/ AIFD and FDD/IFDD, all essential prerequisites to computing coded-transmission performance metrics over correlated channels, are derived in Subsection 3.C. They are confronted by end-to-end simulation results in Section 4, mainly for assessing the accuracy of the underlying physical models, for a LEO as well as a geosynchronous satellite (GEO) downlink scenario. Finally, these statistical and analytical results are exploited in Section 5 by investigating, in a case study, the influence of three distinct $A O$ performances in each scenario (LEO and GEO) on the FDD and IFDD.

\section{MATHEMATICAL DEFINITIONS}

\section{A. Notations}

Bold lowercase and uppercase variables will denote, respectively, vectors and matrices and, throughout this paper, when not used as a subscript, the letter $j$ will denote the imaginary unit. In the following, the operator $\langle\cdot \mid \cdot\rangle_{Z}$ denotes a scalar product with weight $Z$ defined as

$$
\langle X \mid Y\rangle_{Z} \triangleq \iint Z(\mathbf{r}) \cdot X(\mathbf{r}) \cdot Y^{*}(\mathbf{r}) \mathrm{d}^{2} \mathbf{r},
$$

where $X(\mathbf{r}), Y(\mathbf{r}), Z(\mathbf{r}) \in \mathbb{C}$ are functions of the spatial coordinates $\mathbf{r}$, and $Y^{*}(\mathbf{r})$ denotes the complex conjugate of $Y(\mathbf{r})$. 
Following this notation, instantaneous "spatial" normalized averages and variances are defined as

$$
\begin{gathered}
\langle X\rangle_{Z} \triangleq \frac{\langle X \mid 1\rangle_{Z}}{\langle 1 \mid 1\rangle_{Z}}, \\
\sigma_{Z}^{2}(X) \triangleq\left\langle X^{2}\right\rangle_{Z}-\langle X\rangle_{Z}^{2} .
\end{gathered}
$$

This normalization ensures that $\langle 1\rangle_{Z}=1$. The usual statistical average (or time average when time occurrences are considered) as well as statistical variance of the random variable $X(t)$ will be denoted, respectively, as $\mu_{X}$ and $\sigma_{X}^{2}$. The autocovariance of the wide-sense stationary temporal process $V(t)$ is defined as

$$
R_{V}(\tau) \triangleq \operatorname{COV}(V(t), V(t))=E[V(t) V(t+\tau)]-\mu_{V}^{2} .
$$

\section{B. Definitions}

Let us consider an incident beam (unbounded plane wave) after propagation through turbulence and before reaching the telescope pupil of diameter $D$. Its electromagnetic field's complex amplitude is characterized by

$$
\Psi(\mathbf{r}, t)=A_{0} \exp (\chi(\mathbf{r}, t)+j \phi(\mathbf{r}, t)),
$$

where $A_{0}$ denotes the complex field amplitude without perturbation, $\chi(\mathbf{r}, t)$ corresponds to the log-amplitude fluctuations, and $\phi(\mathbf{r}, t)$ represents the phase variations (aberrations). For a perfect plane wave in the absence of turbulence, the incident field would simply be given by $\Psi_{0}(\mathbf{r}, t)=A_{0}$ and, without loss of generality, the normalization of $A_{0}$ is chosen to ensure $\left\langle A_{0} \mid A_{0}\right\rangle_{P}=1$, where $P$ represents the pupil transmittance $P(\mathbf{r})$ defined as

$$
P(\mathbf{r})=\left\{\begin{array}{ll}
1 & \text { if } 0 \leq \frac{2|r|}{D} \leq 1 \\
0 & \text { otherwise }
\end{array} .\right.
$$

The SMF mode expressed in the pupil plane, denoted as $M_{0}$ in the following, is a Gaussian mode whose waist is set in order to maximize its instantaneous matching with the unperturbed incident beam $\Psi_{0}(\mathbf{r}, t)$ [10]. This matching is characterized in the pupil plane by the following normalized overlap integral [11]:

$$
\Omega_{0}=\frac{\left\langle\Psi_{0} \mid M_{0}\right\rangle_{P}}{\left[\left\langle\Psi_{0} \mid \Psi_{0}\right\rangle_{P} \times\left\langle M_{0} \mid M_{0}\right\rangle_{P}\right]^{1 / 2}},
$$

where, for notational convenience, the time dependence of $\Psi_{0}(\mathbf{r}, t)$ and hence $\Omega_{0}(t)$ have been omitted. In the absence of turbulence, the instantaneous coupling efficiency is therefore given by

$$
\rho_{0}=\left|\Omega_{0}\right|^{2} .
$$

Similarly, the matching between the perturbed incident wave $\Psi(\mathbf{r}, t)$ and the SMF mode is given by

$$
\Omega=\frac{\left\langle\Psi \mid M_{0}\right\rangle_{P}}{\left[\langle\Psi \mid \Psi\rangle_{P} \times\left\langle M_{0} \mid M_{0}\right\rangle_{P}\right]^{1 / 2}} .
$$

The squared modulus of the aforementioned overlap integral corresponds to the instantaneous coupling efficiency of the perturbed incident beam,

$$
\rho=|\Omega|^{2},
$$

and hence, the instantaneous coupled flux into the SMF is

$$
f_{\mathrm{SMF}}=|\Omega|^{2} \times\langle\Psi \mid \Psi\rangle_{P}
$$

\section{ANALYTIC MODEL}

In order to properly assess the impact of turbulence on the coupled flux $f_{\mathrm{SMF}}$, both log-amplitude (scintillation) and phase fluctuations (aberrations) must be considered while taking into account partial correction. In the following, this is done analytically as well as by Monte Carlo simulation. An estimator of the coupling efficiency without the impact of scintillation is derived first in Subsection 3.A. The contributions of both scintillation and phase aberrations are taken into account in the derivation of the flux coupled into the SMF in Subsection 3.B, and eventually the analytical statistical properties of the latter are derived in Subsection 3.C.

\section{A. Coupling Efficiency Estimator Neglecting Scintillation}

Neglecting turbulence-induced log-amplitude fluctuations, the electromagnetic field's complex amplitude of the incident beam is given by

$$
\Psi_{\Phi}(\mathbf{r}, t)=A_{0} \exp (j \phi(\mathbf{r}, t)) .
$$

After defining $W_{0}=P M_{0}$, the matching between $\Psi_{\Phi}(\mathbf{r}, t)$ and the SMF mode can be expressed as the following spatial normalized average:

$$
\frac{\Omega_{\Phi}}{\Omega_{0}}=\frac{\left\langle\Psi_{\Phi} \mid M_{0}\right\rangle_{P}}{\left\langle\Psi_{0} \mid M_{0}\right\rangle_{P}}=\langle\exp (j \phi)\rangle_{W_{0}} .
$$

The right-hand term of Eq. (13) can always be written as

$$
\langle\exp (j \phi)\rangle_{W_{0}}=\exp \left(j\langle\phi\rangle_{W_{0}}\right)\left\langle\exp \left[j\left(\phi-\langle\phi\rangle_{W_{0}}\right)\right]\right\rangle_{W_{0}} .
$$

Taking the second multiplicative term of the right-hand side of Eq. (14) in the form of its power series expansion yields

$$
\langle\exp (j \phi)\rangle_{W_{0}}=\exp \left(j\langle\phi\rangle_{W_{0}}\right) \sum_{l=0}^{\infty} \frac{\left\langle\left[j\left(\phi-\langle\phi\rangle_{W_{0}}\right)\right]^{l}\right\rangle_{W_{0}}}{l !} .
$$

By invoking the ergodic hypothesis of the phase, one could assume that it is normally distributed over the pupil. Hence, the moment of order $l$ of $\phi-\langle\phi\rangle_{W_{0}}$ would be given by

$$
\left\langle\left(\phi-\langle\phi\rangle_{W_{0}}\right)^{l}\right\rangle_{W_{0}}= \begin{cases}0 & \text { if } l \text { odd } \\ \sigma_{W_{0}}^{l}(\phi)(l-1) ! ! & \text { if } l \text { even }\end{cases}
$$

and one could write

$$
\langle\exp (j \phi)\rangle_{W_{0}}=\exp \left(j\langle\phi\rangle_{W_{0}}\right) \exp \left(-\frac{\sigma_{W_{0}}^{2}(\phi)}{2}\right) .
$$

However, when the normality of the spatial distribution of the phase over the pupil cannot be justified, Eq. (17) is not exact. Nevertheless, when $\phi-\langle\phi\rangle_{W_{0}} \ll 1$, Ruilier and Cassaing have shown that it could still be a good approximation [5], and Meimon et al. experimentally confirmed it [12]. Therefore, an approximation of the instantaneous normalized coupling efficiency is given by

$$
\rho_{\Phi} \simeq \rho_{0} \exp \left[-\sigma_{W_{0}}^{2}(\phi)\right] .
$$

In theory, over a circular pupil, at a given instant $t$, the turbulent phase $\phi(\mathbf{r})$ can always be expanded on the set constituted by the Zernike polynomials $Z_{i}[9]$ as 


$$
\phi(\mathbf{r})=\sum_{i=1}^{\infty} a_{i} Z_{i}\left(\frac{2 \mathbf{r}}{D}\right),
$$

where $a_{i}$ is the Zernike coefficient associated with the Zernike mode $Z_{i}$. However, in practice, the phase is expanded on a truncated Zernike decomposition constituted of a finite number $N$ polynomials that should be chosen large enough in order to minimize the turbulent wavefront aberrations not taken into account by such a truncation. Moreover, when one considers the coupling of the incident field with an SMF, the set of Zernike polynomials over the weighted pupil does not constitute an orthonormal basis anymore [13]. In that case, by working with Zernike polynomials, one would not be able to take advantage of the properties of a set of polynomials in their orthonormal form. More precisely, the spatial variance of the turbulent phase over the pupil would not be equal to the sum of the squares of the Zernike coefficients (without the piston term) anymore. Dai and Mahajan [14,15] have derived a simple and convenient method that permits the computation of a conversion matrix linking the decomposition coefficients of the Zernike polynomials to the coefficients of a new basis of orthonormal polynomials. For the sake of the self-sufficiency of this paper, it is summarized in Appendix A. Eventually, the orthonormal polynomials' coefficients $\mathbf{b}$ can be obtained from the initial Zernike coefficients a, given the lower triangular conversion matrix $\mathbf{M}$,

$$
\mathbf{b}=\left(\mathbf{M}^{T}\right)^{-1} \mathbf{a},
$$

and the coupling efficiency given by Eq. (18) can be expressed directly in terms of these coefficients,

$$
\rho_{\Phi} \simeq \rho_{0} \exp \left[-\sum_{i=2}^{N} b_{i}^{2}\right] .
$$

Histograms of Monte Carlo computations of the temporal variations of instantaneous coupling efficiency attenuation $\rho_{\Phi}$ are presented in Figs. 1(a) and 1(b) for a GEO as well as a LEO downlink scenario. The link parameters corresponding to both scenarios are detailed in Table 1. Note that the Monte Carlo simulation software used here is different from the end-to-end wave-optics simulator mentioned in the introduction. The former is based upon analytical models that are described in [16] as well. It relies on the computation of random occurrences of centered normally distributed Zernike coefficients for describing the corrected phase. This is done by following an analogous version of the algorithm described in [17], however, without taking into account the nondiagonal terms of the covariance matrix between the Zernike modes. Knowing the Zernike coefficients' temporal spectra [18], the temporal correlation of the residual phase is induced by filtering the temporal power spectra densities of the corrected phase. The residual phase variance characterizing the performance of the simulated $\mathrm{AO}$ system comprises terms related to the finite number of actuators (fitting error), wavefront sensing precision (aliasing error) and control-loop frequency (temporal error). In Fig. 1, these simulated residual phase occurrences were used for assessing the accuracy of the approximations given by the squared modulus of Eq. (15) as well as Eqs. (18) and (21) through

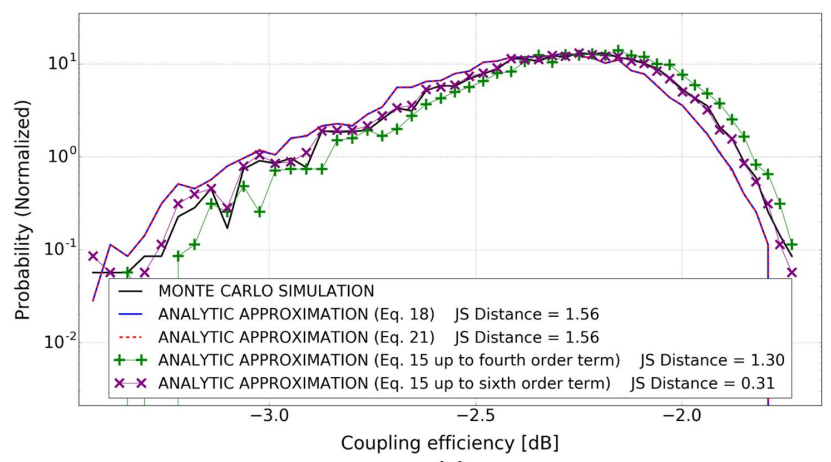

(a)

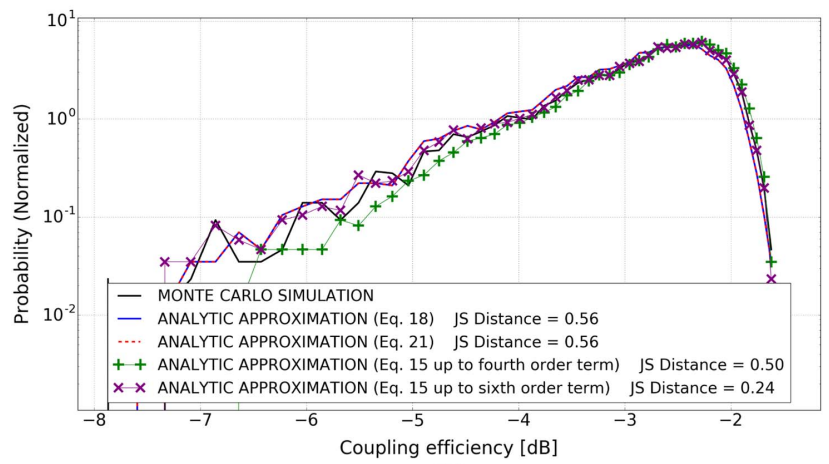

(b)

Fig. 1. Histograms of Monte Carlo simulated coupling efficiency for (a) GEO-to-ground downlink scenario and (b) LEO-to-ground downlink scenario.

the computation of their respective Jensen-Shannon (JS) distances [19] with the histogram of $\rho_{\Phi}$.

The results corresponding to Eqs. (18) and (21) are perfectly equivalent, and hence validate the reorthonormalization process of the Zernike polynomials weighted by the Gaussian mode of the SMF. For comparison, the expansion in Eq. (15) is carried up to the fourth and sixth orders as well by computing the corresponding $l$ th central moment of the phase using the orthonormal polynomial decomposition approach:

$$
\left\langle\left[j\left(\phi-\langle\phi\rangle_{W_{0}}\right)\right]^{l}\right\rangle_{W_{0}}=j \sum_{j=2}^{N} \cdots \sum_{k=2}^{N} b_{j} \cdots b_{k}\left\langle F_{j} \cdots F_{k}\right\rangle_{W_{0}} .
$$

\begin{tabular}{|c|c|c|c|c|}
\hline \multicolumn{3}{|c|}{ Downlink Scenario } & GEO & LEO \\
\hline \multicolumn{3}{|l|}{ Elevation } & $30 \mathrm{deg}$ & $20 \mathrm{deg}$ \\
\hline \multicolumn{3}{|l|}{ Range } & $38,000 \mathrm{~km}$ & 1584 km \\
\hline \multicolumn{3}{|l|}{ Orbital velocity } & $3.0 \mathrm{~km} \cdot \mathrm{s}^{-1}$ & $7.5 \mathrm{~km} \cdot \mathrm{s}^{-1}$ \\
\hline \multicolumn{3}{|c|}{$\mathrm{Rx}$ aperture diameter } & $50 \mathrm{~cm}$ & $25 \mathrm{~cm}$ \\
\hline \multicolumn{3}{|c|}{ Fried parameter $r_{0}$} & $0.069 \mathrm{~m}$ & $0.056 \mathrm{~m}$ \\
\hline \multicolumn{3}{|c|}{ Log-amplitude variance $\sigma_{\chi}^{2}$} & 0.067 & 0.135 \\
\hline \multirow{4}{*}{$\mathrm{AO}$ parameters } & $N_{r}$ & 9 & 6 & \\
\hline & $f_{s}$ & $1 \mathrm{kHz}$ & $2 \mathrm{kHz}$ & \\
\hline & $\sigma_{\text {res }}^{2}$ & 0.4 & 0.5 & \\
\hline & $\mu_{f_{\mathrm{SMF}}}$ & $-2.7 \mathrm{~dB}$ & $-3.2 \mathrm{~dB}$ & \\
\hline
\end{tabular}

Table 1. Characteristic Link, Turbulent, and AO Parameters for the GEO-to-Ground and LEO-to-Ground Downlink Scenarios 
This requires the expensive calculation of $l$ nested sums of the products of $l$ polynomials and their expansion coefficients and quickly leads to intractable analytical statistical results. Owing to the nonnormality of the phase distribution over the pupil, carrying the expansion in Eq. (15) up to the sixth order yields more accurate results than Eq. (18). On the other hand, while providing a simpler expression, Eq. (21) yields results as precise as a fourth-order expansion, hence justifying the choice made in the following analytical developments to use the former instead of a slightly more precise expression related to Eq. (22). This is true at least for the two cases presented here. Finally, note that the accuracy of such Monte Carlo simulations depends on the finite number of modes representing the turbulent wavefront, which, as it gets larger, requires a higher number of pixels describing the pupil for proper sampling. Accurate results, depending on the scenario and turbulent conditions under consideration, hence may come at the expense of computing time that can be comparable to those related to end-to-end wave-optics-based simulations, thus motivating the recourse to precise analytical expressions.

\section{B. Coupled Flux}

In the ideal case, where the phase fluctuations induced by the atmosphere are completely corrected (or otherwise neglected), it is shown in Appendix B that by using the results of [20], the statistical average of the coupled flux thus impacted only by scintillation can be approximated by

$$
\begin{aligned}
\mu_{f_{\text {SMF }}^{\text {sint }}} \simeq & \exp \left(-C_{\chi}(0)\right) \\
& \times\left(1+\frac{\int \mathcal{F}\left[C_{\chi}(\mathbf{r})\right](\omega)\left[\mathcal{F}\left[W_{0}(\mathbf{r})\right](\omega)\right]^{2} \mathrm{~d}^{2} \omega}{\rho_{0} \times\langle 1 \mid 1\rangle_{P}}\right),
\end{aligned}
$$

where $C_{\chi}(\mathbf{r})$ is the log-amplitude spatial covariance function. In this equation, the numerator of the last term on the righthand side corresponds to the spectral power density of the logamplitude fluctuations in the pupil filtered by the instrument (that is the pupil and the SMF) and represents the influence of the spatial correlation of the speckles in the pupil on the mean coupled flux attenuation. Because the difference has been verified numerically to be negligible for the downlink scenarios presented in the remainder of this paper, it is approximated by the spectral power density of the log-amplitude fluctuations filtered by the pupil only. Furthermore, as long as one does not consider strong fluctuation regimes of $\chi(\mathbf{r}, t)$, it is widely accepted that the aperture-averaged irradiance fluctuations are $\log$-normally distributed $[21,22]$. The random generation of the latter is done using an algorithm analogous to the one described in [23]. These irradiance fluctuations, denoted $\exp \left(2 \chi_{\mathrm{Ap}}(t)\right)$ in the following, depends on the Gaussian random variable $\chi_{\mathrm{Ap}}(t)$, representing the aperture-averaged logamplitude fluctuations that are characterized by their variance $\sigma_{\chi_{\mathrm{Ap}}}^{2}$. The analytic model describing this variable, including its temporal power spectrum, can be found in [24].

The log-normality assumption of the aperture-averaged irradiance can be used to state that in this regime the completely corrected coupled flux into the SMF can be described as well by a log-normal random variable. By setting the mean of the aperture-averaged log-amplitude fluctuations equal to
$-\sigma_{\chi_{\mathrm{Ap}}}^{2}$, it is ensured that $\mu_{\exp \left(2 \chi_{\mathrm{Ap}}\right)}=1$. Thence, the random variable $\mu_{f_{\mathrm{SMF}}^{\text {sint }}} \times \exp \left(2 \chi_{\mathrm{Ap}}(t)\right)$ represents the scintillation-only induced variations of the coupled flux, whose mean is fixed by Eq. (23). By considering the different origins of the fluctuations of the log-amplitude and the phase (that is, the former is mostly caused by distant turbulence, whereas the latter is mostly introduced close to ground [25]), one can assume statistical independence of scintillation and phase effects. Therefore, after combining the results characterizing the completely corrected case described above and those of Subsection 3.A, the instantaneous coupled flux in an SMF after partial $\mathrm{AO}$ correction is approximated by

$$
f_{\mathrm{SMF}}(t) \simeq \rho_{0} \mu_{f_{\mathrm{SMF}}^{\text {sint }}} \exp \left(2 \chi_{\mathrm{Ap}}(t)\right) \exp \left[-\sum_{i=2}^{N} b_{i}(t)^{2}\right] .
$$

For the sake of clarity, hereafter the principle of the Monte Carlo process developed to generate temporally correlated random fluctuations of the coupled flux is summarized. The random generation of aperture-averaged irradiance fluctuations is implemented as in [23] and assumes weak-to-moderate turbulence conditions. These fluctuations are temporally correlated by filtering them by the spectrum described in [24]. The impact of scintillation on the coupled flux is statistically taken into account on average, thanks to Eq. (23). Phase fluctuations are initially generated thanks to an algorithm close to the one described in [17], which simulates atmospherically distorted wavefronts using a Zernike expansion. The temporal correlation of the residual phase is induced by filtering the Zernike coefficients according to the temporal spectra given in [18]. Injection efficiency fluctuations are obtained by directly computing the weighted scalar product between the residual phase and the Gaussian mode of the SMF. The analytical approximation counterpart of the injection efficiency is obtained after converting the correlated Zernike coefficients into orthonormal polynomials coefficients [see Eq. (20)] and using the approximation given by Eq. (21). Eventually, assuming statistical independence between scintillation and phase effects, the product of the aperture-averaged irradiance random variables with those of the injection efficiency yields random variables of coupled flux. Analytically, this is approximated by Eq. (24), for which statistical laws are found next.

\section{Statistical Properties of the Coupled Flux}

\section{Statistical Distribution Functions: PDF and CDF}

Let us first derive the PDF and CDF of the quantity $\sum_{i=2}^{N} b_{i}^{2}(t)$. Since the Zernike coefficients $\left\{a_{i}\right\}$ are Gaussian random variables [26], in light of Eq. (20), it is clear that the coefficients $\left\{b_{i}\right\}$ are Gaussian as well. Therefore, the square of each one of the latter is gamma-distributed with constant shape parameter $\alpha=0.5$ and scale parameter $\beta_{i}=2 \sigma_{b_{i}}$, where $\sigma_{b_{i}}$ is the statistical variance of the coefficient corresponding to mode $i$. In consequence, the sum of these coefficients $\sum_{i=2}^{N} b_{i}^{2}(t)$ is characterized by a sum of independent and nonidentical gamma random variables. Note that in light of Eq. (20), it might be argued that the coefficients $\left\{b_{i}\right\}$ are not completely independent. However, it is shown in Appendix A that $\left(\mathbf{M}^{T}\right)^{-1}$ is nearly diagonal, and hence, the dependence between the aforementioned coefficients is negligible. 
The marginal characteristic function of such a process can be written as

$$
\varphi(u)=\prod_{i=2}^{N}\left(1-\frac{j u}{\beta_{i}}\right)^{-\alpha} .
$$

By performing the inverse Laplace transform of this characteristic function, the PDF of $z(t)=\sum_{i=2}^{N} b_{i}^{2}(t)$ can be obtained using Gil-Pelaez's result [27]:

$$
p_{Z}(z)=\frac{1}{\pi} \int_{0}^{\infty} \frac{\cos \left(\sum_{i=2}^{N} \alpha \arctan \left(\beta_{i} u\right)-z u\right)}{\prod_{i=2}^{N}\left(1+u^{2} \beta_{i}^{2}\right)^{\frac{\alpha}{2}}} \mathrm{~d} u .
$$

The CDF can be determined as well:

$$
P_{Z}(z)=\frac{1}{2}-\frac{1}{\pi} \int_{0}^{\infty} \frac{\operatorname{Im}\left\{\prod_{i=2}^{N}\left(1-\beta_{i j} j u\right)^{-\alpha} \exp [-j u z]\right\}}{u} \mathrm{~d} u \text {. }
$$

To the best of our knowledge, this result was first published by Efthymoglou and Aalo [28]. More recently, several authors have presented alternative forms characterizing the distribution of such random variables $[29,30]$. However, although they may seem more tractable, these solutions can be more difficult to compute numerically. Using a simple change of variable, the $\mathrm{PDF}$ and $\mathrm{CDF}$ of the normalized coupling efficiency given by Eq. (21) are, respectively,

$$
\begin{gathered}
p_{P_{\Phi}}\left(\rho_{\Phi}\right)=\frac{1}{\rho_{\Phi}} p_{Z}\left(\log \left(\frac{\rho_{0}}{\rho_{\Phi}}\right)\right), \\
P_{P_{\Phi}}\left(\rho_{\Phi}\right)=1-P_{Z}\left(\frac{\rho_{\Phi}}{\rho_{0}}\right),
\end{gathered}
$$

deriving the channel capacity and probability of outages of freespace optical (FSO) transmissions [31].

\section{Fade Duration Distribution}

Temporal autocovariance approximation: Let us first derive an approximation of the autocovariance of the coupling efficiency $\rho_{\Phi}(t)$ given by Eq. (21). It is by definition a wide-sense stationary random process. The first-order expansion of its autocovariance is hence given by

$$
R_{\rho_{\Phi}}(\tau) \simeq \rho_{0}^{2}\left(E\left[\sum_{i=2}^{N} b_{i}^{2}(t) \sum_{i=2}^{N} b_{i}^{2}(t+\tau)\right]-\mu_{\sum_{i=2}^{N} b_{i}^{2}}\right) .
$$

Since every $\left\{b_{i}\right\}$ is a centered normal random variable, $\mu_{\sum_{i=2}^{N} b_{i}^{2}}^{2}=\left(\sum_{i=2}^{N} \sigma_{b_{i}}^{2}\right)^{2}$. By assuming that intermodal covariances are negligible when compared to the modal covariances and by subsequently using the theorem of Isserlis [32,33], allowing one to express higher-order moments of Gaussian random variables in terms of their covariances, it becomes

$$
R_{\rho_{\Phi}}(\tau) \simeq 2 \rho_{0}^{2} \sum_{i=2}^{N} E\left[\left(b_{i}(t) b_{i}(t+\tau)\right]^{2} .\right.
$$

A simple approximation of the autocovariance of the coupling efficiency $\rho_{\Phi}(t)$ is therefore obtained, since the analytical models describing the Zernike coefficients temporal spectra are well known [18], whereas Zernike's interspectrum can be obtained as in [34]. Figure 2 compares the autocorrelation functions corresponding to the approximations given by Eqs. (32) and (33) and the Monte Carlo simulations. Bartlett's 95\% confidence intervals are shown as well [35]. For both LEO and GEO links, the first-order approximation of the instantaneous

$$
\begin{gathered}
p_{F_{\mathrm{SMF}}}\left(f_{\mathrm{SMF}}\right)=\frac{1}{\pi \sqrt{2 \pi} \sigma_{I} f_{\mathrm{SMF}}} \int_{-\infty}^{\infty}\left[\int_{0}^{\infty} \frac{\cos \left(\sum_{i=2}^{N} \alpha \arctan \left(\beta_{i} v\right)-\ln \left(\frac{\rho_{0} \mu_{f} \text { Sint }}{u}\right) v\right)}{\prod_{i=2}^{N}\left(1+v^{2} \beta_{i}^{2}\right)^{\frac{\alpha}{2}}} \mathrm{~d} v\right] \frac{1}{|u|} \exp \left(-\frac{\left[\ln \left(\frac{f_{\mathrm{SMF}}}{u \mu_{i}}\right)+\frac{\sigma_{I}^{2}}{2}\right]^{2}}{2 \sigma_{I}^{2}}\right) \mathrm{d} u \\
P_{F_{\mathrm{SMF}}}\left(f_{\mathrm{SMF}}\right)=\int_{0}^{\infty}\left[\frac{1}{2}+\frac{1}{2} \operatorname{erf}\left(\frac{\ln \left(\frac{f_{\mathrm{SMF}}}{u \mu_{I}}+\frac{\sigma_{I}^{2}}{2}\right)}{\sqrt{2} \sigma_{I}}\right)\right] \frac{1}{u \pi}\left[\int_{0}^{\infty} \frac{\cos \left(\sum_{i=2}^{N} \alpha \arctan \left(\beta_{i} v\right)-\ln \left(\frac{\rho_{0} \mu_{f} f_{\mathrm{SMF}}}{u}\right) v\right)}{\prod_{i=2}^{N}\left(1+v^{2} \beta_{i}^{2}\right)^{\frac{\alpha}{2}}} \mathrm{~d} v\right] \mathrm{d} u
\end{gathered}
$$

On the basis of the assumption of the independence of logamplitude and phase fluctuations, the PDF and the CDF of the random variable defined by Eq. (24) are given, respectively, by Eqs. (30) and (31), in which $\sigma_{I}^{2}$ is the aperture-averaged logirradiance variance (approximated by the scintillation index in the weak fluctuation regime) and $\mu_{I}=\mu_{\exp \left(2 \chi_{\mathrm{Ap}}\right)}=1$ (see Subsection 3.B). These analytical PDFs and CDFs of the instantaneous coupled flux attenuation are, per se, useful results in coupling efficiency yields fairly accurate results. Neglecting intermodal correlations provides a less accurate approximation that is, nevertheless, still inside the confidence bands. The errors on the correlation time (width at half-maximum) are inferior to $3 \%$. Using the result of [36] dealing with the autocovariance of the product of two stationary time series, the autocovariance of the coupled flux after partial $\mathrm{AO}$ correction is given by 


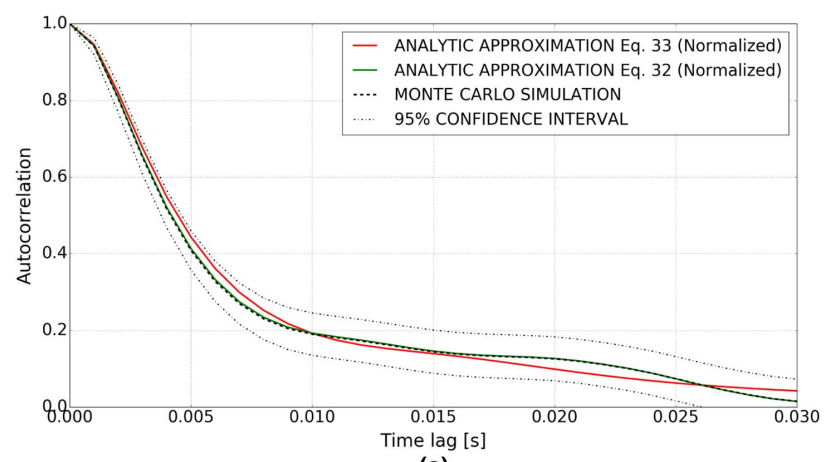

(a)

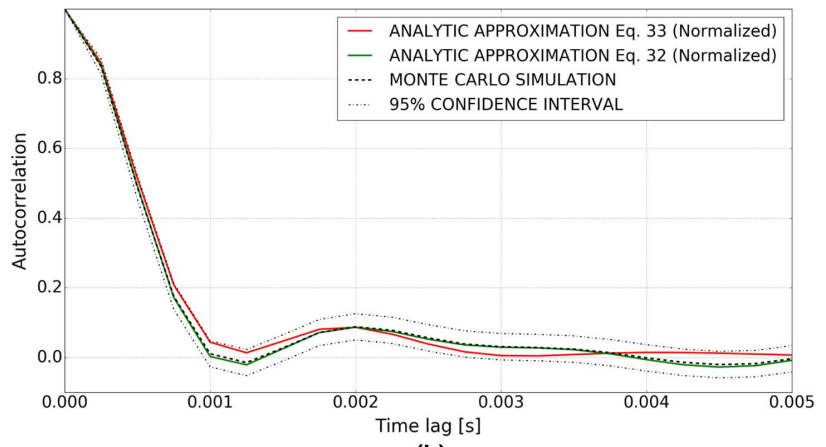

(b)

Fig. 2. Numerically computed autocorrelation functions of Monte Carlo simulation results for (a) GEO-to-ground downlink scenario and (b) LEO-to-ground downlink scenario.

$$
\begin{aligned}
R_{f_{\mathrm{SMF}}}(\tau)= & \mu_{f_{\mathrm{SMF}}}^{2 \text { sint }}\left[R_{\rho_{\Phi}}(\tau) R_{\exp \left(2 \chi_{A p}\right)}(\tau)\right. \\
& \left.+\mu_{\rho_{\Phi}}^{2} R_{\exp \left(2 \chi_{A p}\right)}(\tau)+R_{\rho_{\Phi}}(\tau)\right] .
\end{aligned}
$$

As mentioned in Subsection 3.B, the aperture-averaged irradiance's temporal characteristics are modeled as in [24], and $\mu_{\rho_{\Phi}}$ can be obtained thanks to the PDF given by Eq. (28).

Average level-crossing rate and average fade duration: For an arbitrary stationary differentiable random process such as $f_{\mathrm{SMF}}(t)$ with a given $\operatorname{PDF} p_{F_{\mathrm{SMF}}}\left(f_{\mathrm{SMF}}\right)$, it is possible to compute an approximation of the average unidirectional (upward or downward) level-crossing rate $\nu\left(f_{\mathrm{SMF}}^{0}\right)$ at a given threshold $f_{\mathrm{SMF}}^{0}$ by using the result of [37],

$$
\nu\left(f_{\mathrm{SMF}}^{0}\right)=\frac{1}{2} \frac{\sigma_{\dot{f}_{\mathrm{SMF}}}}{\pi \sqrt{\gamma}} \exp -\frac{h^{2}\left(f_{\mathrm{SMF}}^{0}\right)}{2},
$$

where $\sigma_{\dot{f}_{\mathrm{SMF}}}$ is the variance of the time derivative of $f_{\mathrm{SMF}}(t)$, which is, by definition [38], given by the second time derivative of the autocovariance function in the vicinity of 0 ,

$$
\sigma_{\dot{f}_{\mathrm{SMF}}}^{2}=-\left.\ddot{R}_{f_{\mathrm{SMF}}}(\tau)\right|_{\tau=0} .
$$

The function $h($.$) depends on the \mathrm{CDF}$ of $f_{\mathrm{SMF}}(t)$, i.e., $P_{F_{\text {SMF }}}\left(f_{\text {SMF }}\right)$ given by Eq. (31),

$$
h\left(f_{\mathrm{SMF}}^{0}\right)=\sqrt{2} \operatorname{erf}^{-1}\left(2 P_{F_{\mathrm{SMF}}}\left(f_{\mathrm{SMF}}^{0}\right)-1\right),
$$

whereas $\gamma$ depends on the PDF of $f_{\mathrm{SMF}}(t)$, i.e., $p_{F_{\mathrm{SMF}}}\left(f_{\mathrm{SMF}}\right)$ given by Eq. (30),

$$
\gamma=\int_{F_{\mathrm{SMF}}} \frac{p_{F_{\mathrm{SMF}}}\left(f_{\mathrm{SMF}}\right)}{\left[\frac{d h}{d f_{\mathrm{SMF}}}\right]^{2}} \mathrm{~d} f_{\mathrm{SMF}} .
$$

The average fade duration under the threshold $f_{\text {SMF }}^{0}$ is finally equal to

$$
\bar{\tau}_{\text {fade }}\left(f_{\text {SMF }}^{0}\right)=\frac{P_{F_{\mathrm{SMF}}}\left(f_{\mathrm{SMF}}^{0}\right)}{\nu\left(f_{\mathrm{SMF}}^{0}\right)} .
$$

The average interfade duration corresponding to threshold $f_{\mathrm{SMF}}^{0}$ is obtained by replacing the CDF $P_{F_{\mathrm{SMF}}}\left(f_{\mathrm{SMF}}^{0}\right)$ in the previous equation by the complementary cumulative distribution $1-P_{F_{\mathrm{SMF}}}\left(f_{\mathrm{SMF}}^{0}\right)$.

Fade duration distribution: The AFD is an important parameter for the design of efficient numerical fading-mitigation techniques such as interleaving and forward error-correction codes on bursty channels. The AFD can be used to infer the distribution of the fades' duration, the latter being essential to the evaluation of coded error probability on correlated fading channels without interleaving or with imperfect interleaving [39-41]. In [42], these distributions were obtained from experimental links data and compared to those generated by a twostate Markov (Gilbert-Erasure) model. This kind of model was also validated through experimentation for the atmospheric channel in $[43,44]$. Assuming the Markovian nature of the fading process as well, a similar two-state discrete time Markov model is used here to analytically compute the exceedance probability of fades' duration (i.e., the probability of facing a fade duration greater than the one considered) of the SMFcoupled flux after partial $\mathrm{AO}$ correction:

$$
\operatorname{Pr}\left(\tau_{\text {fade }}>\tau_{\text {fade }}^{0}\right)=\left(1-\frac{1}{\bar{\tau}_{\text {fade }}\left(f_{\text {SMF }}^{0}\right)}\right)^{\tau_{\text {fade }}^{0}} .
$$

Interfade duration distributions are computed by replacing the AFD $\bar{\tau}_{\text {fade }}\left(f_{\text {SMF }}^{0}\right)$ by the AIFD corresponding to the same threshold $f_{\mathrm{SMF}}^{0}$.

\section{COMPARISON TO END-TO-END WAVE- OPTICS SIMULATIONS}

The analytic results described in Subsection 3.C are hereafter compared to end-to-end wave-optics simulation results for a GEO-to-ground as well as a LEO-to-ground link. This endto-end simulation software has been previously validated experimentally $[45,46]$. The transmission wavelength is $1.55 \mu \mathrm{m}$. The turbulence and wind profiles used here are given in [16]. The parameters characterizing the simulated AO systems, such as the number of corrected radial orders, $N_{r}$ the control loop frequency $f_{s}$, and the residual phase variance $\sigma_{\text {res }}^{2}$, as well as other link parameters, are given in Table 1. Both links are representative of an $\mathrm{AO}$ performance level corresponding to a mean coupled flux attenuation of roughly $3 \mathrm{~dB}$.

The results obtained from the simpler numerical tool based on a Monte Carlo approach introduced in Subsection 3.A and, based on the analytic framework described in Subsection 3.B for the treatment of scintillation effects, are presented as well. Figures 3(a) and 3(b) show the residual phase spectra, respectively, for the GEO and LEO links. Partial AO is characterized by the combined effect of fitting, aliasing, and temporal errors of the loop. For every system simulated in this paper, 


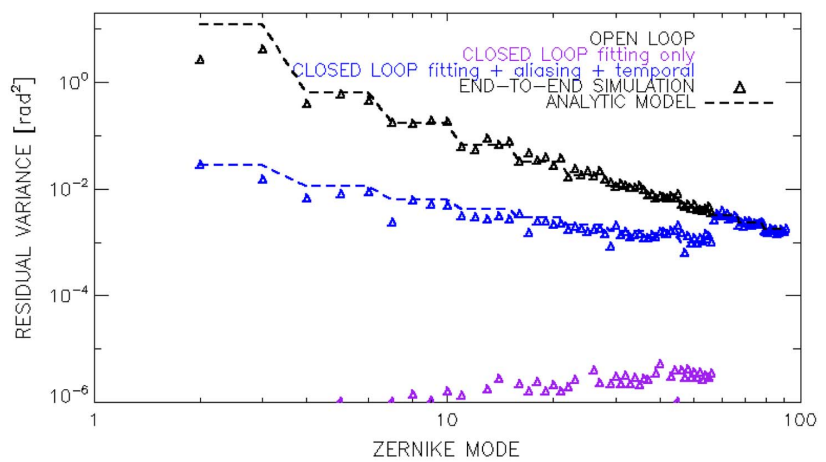

(a)

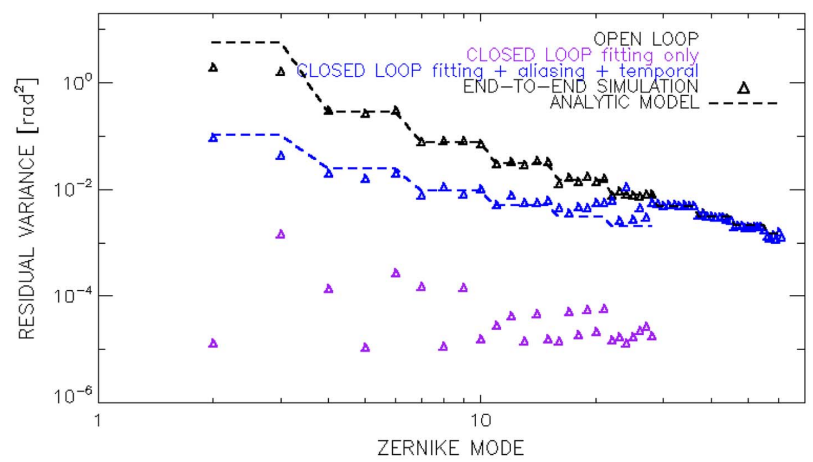

(b)

Fig. 3. Residual modal variances obtained by end-to-end waveoptics simulations as well as analytic models for (a) GEO-to-ground downlink scenario and (b) LEO-to-ground downlink scenario.

the proportion of temporal errors equals the proportion of spatial error (fitting error plus aliasing error). In both figures, a good agreement between the predicted analytic residual variances and the end-to-end simulations can be observed. It should be noted, however, that in both Monte Carlo simulation and analytic results, the influence of noise and scintillation on wavefront sensing has been neglected. In the end-to-end simulation, the wavefront sensor noise is ignored, but the impact of scintillation is taken into account in the modeling process of the images in the focal plane of the wavefront sensor. As evidenced by the point-specific log-amplitude variances in Table 1, scintillation under these weak turbulence conditions negligibly affects the accuracy of wavefront measurement $[26,47]$. This is especially true, since the influence of noise on the wavefront sensor is neglected. In the presence of significant noise-for cases of low signal-to-noise ratio-this analysis should be reconsidered. However, given the typical optical power required for high data-rate satellite-to-ground communication [48], the signal levels required for the wavefront analysis remain very reasonable and allow one to consider high signal-to-noise ratios. To fine-tune the analysis, consideration should be given to the limited dynamics of the sensor, the flux allocated to the wavefront analyzer, the sensitivity of the sensors used, as well as the slope calculation algorithms.

\section{A. Statistical Results: GEO-to-Ground Scenario}

First, in order to validate Eq. (23), let us consider the case of a full and perfect correction of the turbulent wavefront or, in

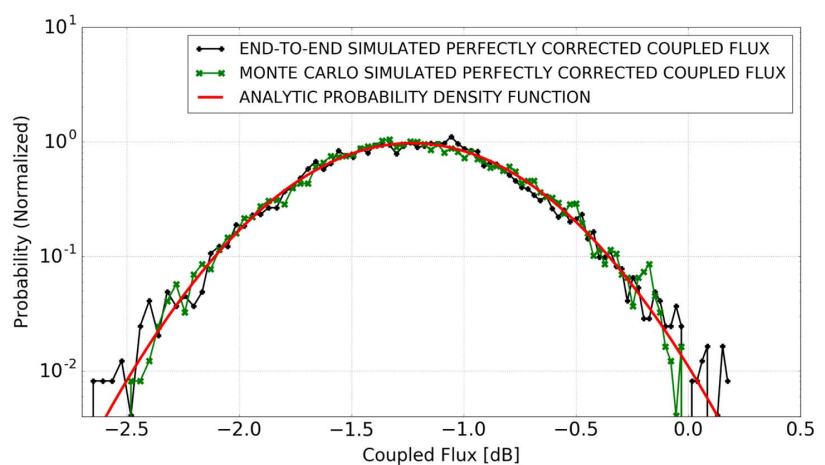

(a)

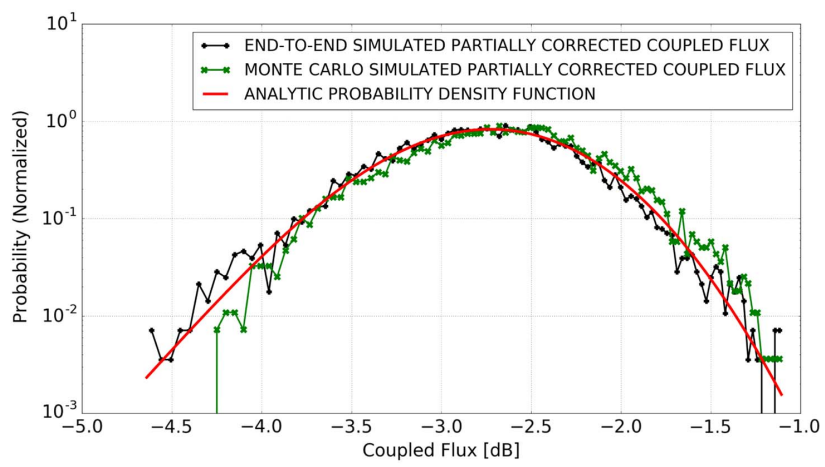

(b)

Fig. 4. Comparison of the analytical PDF to end-to-end as well as Monte Carlo simulations histograms of (a) coupled flux without phase fluctuations (fully compensated turbulent wavefront) and (b) partially corrected coupled flux for the GEO scenario.

other words, neglect phase fluctuations. In that case, the coupled flux attenuation is given by Eq. (24) with the sum of the modal coefficients in the last term being equal to zero and therefore follows a log-normal distribution. Figure 4(a) shows the corresponding analytic density and the simulated (end-to-end and Monte Carlo) distributions. In order to ensure stochastic convergence, a temporal series of 8000 samples with a correlation time of $7.3 \mathrm{~ms}$ over a time horizon of $8 \mathrm{~s}$ was generated. For the end-to-end simulation, 26 phase screens of $3.3 \mathrm{~m}$ and 512 pixels were simulated. This corresponds to a $6.4 \mathrm{~mm}$ pixel size, small enough to appropriately sample the Fried parameter. The absolute difference between the endto-end simulated and theoretical average coupled flux attenuation is less than $0.1 \%$. Furthermore, Kolmogorov-Smirnov (KS) goodness-of-fit tests [49,50] performed between the analytical and empirical CDFs yielded statistically conclusive results, hence confirming the relevance of the approach regarding the treatment of the log-amplitude fluctuations' effect on the coupled flux in that case. The distributions corresponding to both the effect of scintillation and phase fluctuations on the coupled flux are presented in Fig. 4(b). Due to the numerical precision variability of the Monte Carlo-based simulation discussed in Subsection 3.A, the latter provides results that differ slightly from the end-to-end results. Regarding the average coupled power and its coefficient of variation, the aforementioned discrepancies are about 5\%, depending on the precision desired and hence, at the expense of computing time. 
The analytic model, statistically validated anew by goodness-offit tests, on the other hand, yields fairly accurate results. While there are differences on the order of $0.5 \%$ and $3 \%$ when comparing, respectively, the first two moments of the analytic and end-to-end distributions, these differences stem from the statistical convergence of such estimators and comprise the standard errors related to their accuracy [51,52]. Consequently, for the GEO-to-ground scenario considered here, where partial $\mathrm{AO}$ as well as scintillation effects are considered, our analytic model adequately captures the statistical behavior of the transmission channel. The temporal characteristics of the latter are, as mentioned earlier, important as well in optimizing coding strategies. For instance, the AFD/AIFD and FDD/IFDD are required for the computation of higher layer packet error rates while considering an imperfect interleaving of the channel $[40,53]$. An important parameter for the computation of the AFD and AIFD is the value of the second time derivative of the autocovariance (i.e., a measure of its concavity) at zero time lag, $\left.R_{f_{\text {SMF }}}(\tau)\right|_{\tau=0}$. Hereinafter, the evolution of the autocovariance of the coupled flux against time lag obtained by both simulation models as well as the analytic approximation given by Eq. (34) is presented in Fig. 5. The overall good agreement between our analytic results and simulations models regarding the autocovariance as well as the probability distribution of the coupled flux allow for a fairly precise prediction of the evolution of the AFD and AIFD with respect to the fading/interfading threshold, as presented in Figs. 6(a) and 6(b). From an overall system point of view, relevant fading levels depend on the link budget as well as the detection and modulation/coding schemes considered. Nevertheless, for the GEO-to-ground scenario presented here, one can see that the AFD corresponding to the mean level fading threshold $(-2.73 \mathrm{~dB})$ is about $10 \mathrm{~ms}$ (it is roughly equal to the AIFD for this threshold, as expected) and that it is divided by a factor of 3 when the mean level fading threshold is roughly $-4.5 \mathrm{~dB}$. Figures $7(\mathrm{a})$ and 7 (b) show, respectively, the end-to-end FDD and IFDD for the mean level fading threshold. A fine agreement between these distributions and the analytic geometric law described by Eq. (40) can be observed. KS goodness-of-fit tests have been performed, and the geometric densities are well inside the $95 \%$ confidence bands. Combined with the experimental results presented in [42], this tends to corroborate the hypothesis that two-state Markov channels could emulate the correlated burst-like fades

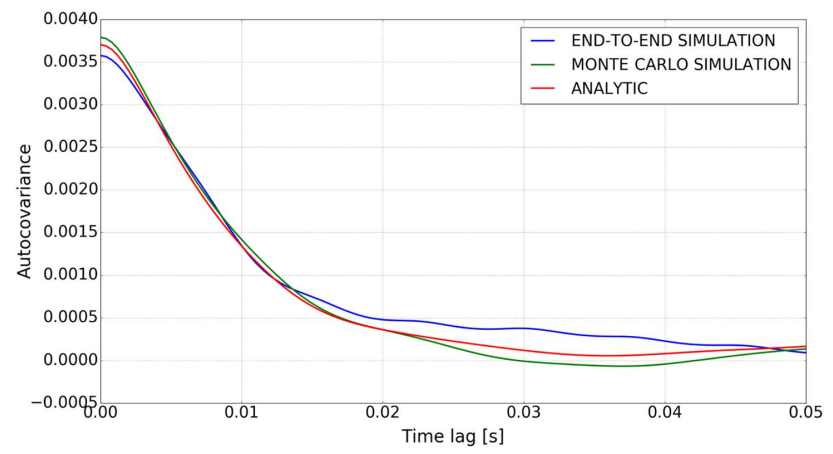

Fig. 5. Autocovariance of the partially corrected coupled flux given by the analytic approximation as well as the end-to-end and Monte Carlo simulations for the GEO scenario.

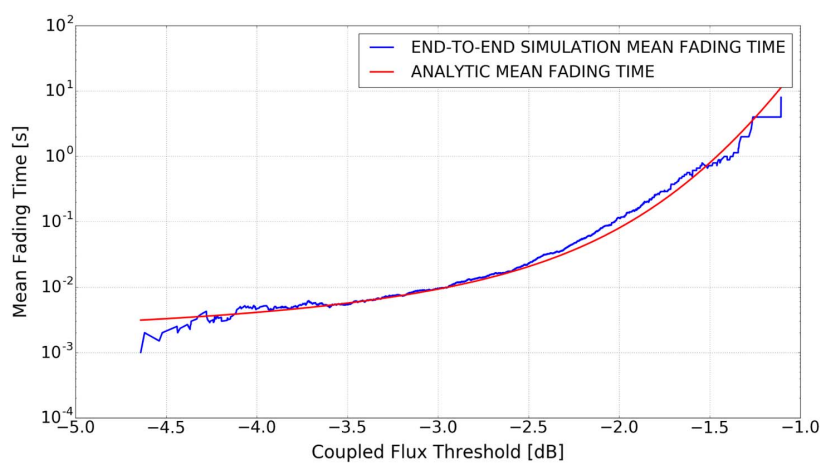

(a)

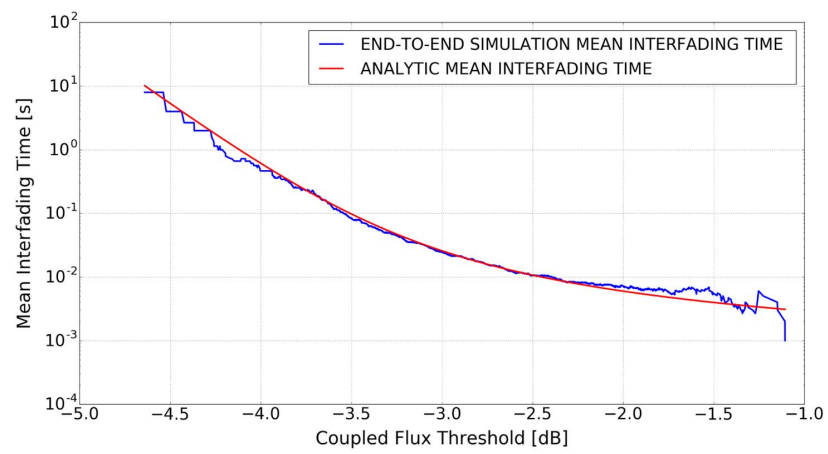

(b)

Fig. 6. Comparison of the evolution against the fading threshold of analytic and end-to-end (a) average fade duration and (b) average interfade duration for the GEO scenario.

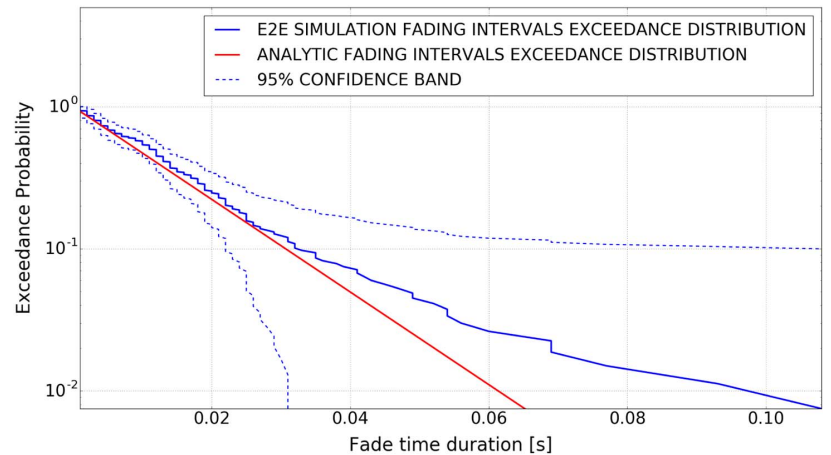

(a)

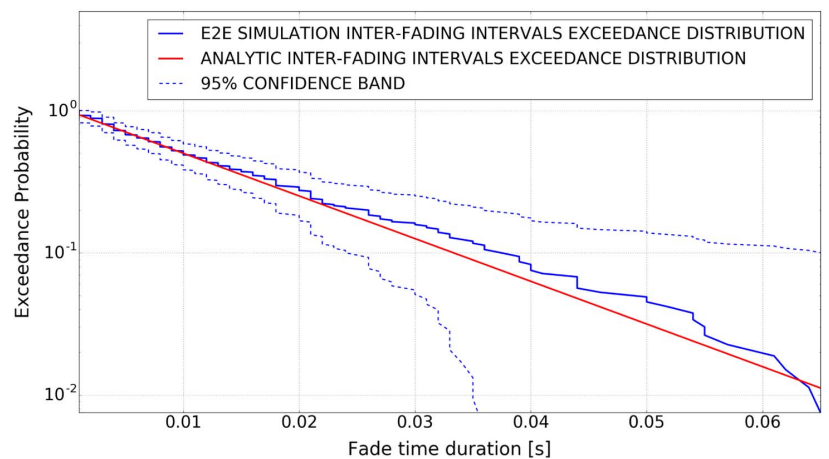

(b)

Fig. 7. Comparison of the analytic and end-to-end exceedance distributions of (a) fade duration and (b) interfade duration for a fading threshold corresponding to the average coupled flux attenuation for the GEO scenario. 
induced by turbulence over the free-space optical channel. Our results extend the relevance of such modeling options to the case of AO-corrected SMF coupled signals for the GEO downlinks and will therefore permit an accurate and rigorous joint optimization of $\mathrm{AO}$ systems and numerical mitigation techniques of interest.

\section{B. Statistical Results: LEO-to-Ground Scenario}

A similar analysis is done in the LEO scenario, for which a temporal series $2 \mathrm{~s}$ long, with 8000 occurrences and a correlation time of $0.6 \mathrm{~ms}$, was generated. For the end-to-end simulation, 36 phase screens of $2.13 \mathrm{~m}$ and 256 pixels were simulated. This corresponds to an $8.3 \mathrm{~mm}$ pixel size, small enough to appropriately sample the Fried parameter. Figure 8 (a) shows the distribution of the coupled flux when phase fluctuations are completely compensated. The absolute relative difference between the end-to-end simulated and predicted theoretical average attenuation is around $0.3 \%$. The distributions of the partially corrected attenuation are presented in Fig. 8(b). Analogous to the GEO case, if reasonable computation times are required, the Monte Carlo simulations are less precise than the analytic results. The latter were, again, statistically validated by KS tests at a $95 \%$ confidence level. The difference in terms of average coupled flux is less than $1 \%$. However, one can deduce from the autocovariances presented in Fig. 9 that the absolute difference between the analytically and end-toend computed variances is about $8 \%$. This discrepancy is

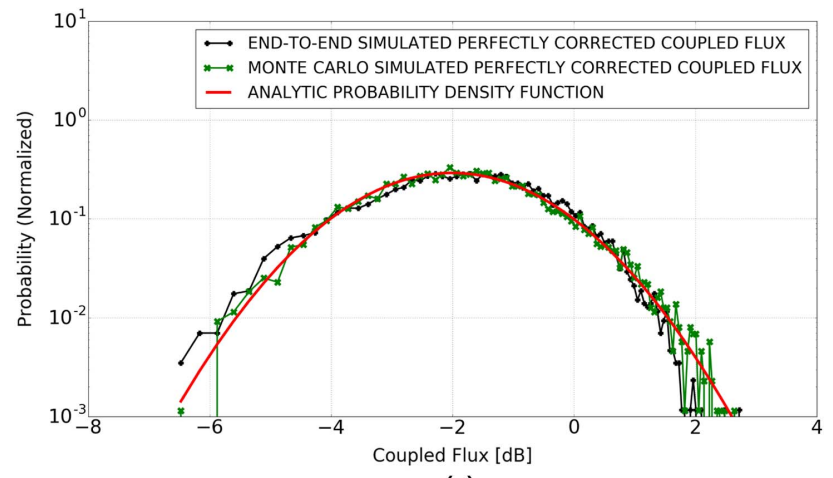

(a)

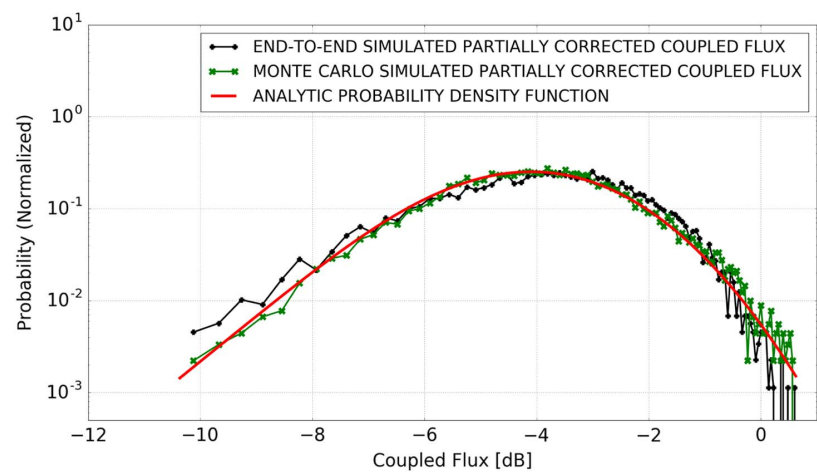

(b)

Fig. 8. Comparison of the analytical PDF to end-to-end as well as Monte Carlo simulations histograms of (a) coupled flux without phase fluctuations (fully compensated turbulent wavefront) and (b) partially corrected coupled flux for the LEO scenario.

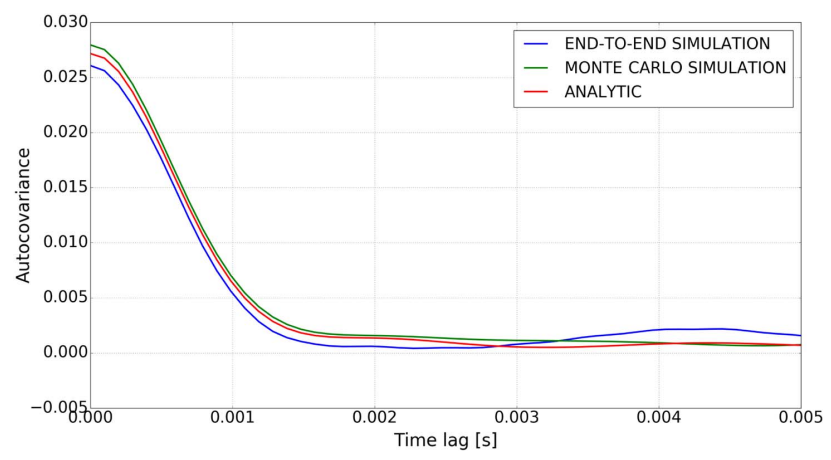

Fig. 9. Autocovariance of the partially corrected coupled flux given by the analytic approximation as well as the end-to-end and Monte Carlo simulations for the LEO scenario.

statistically relevant, since the standard error related to the accuracy of the sample variance is only about $5 \%$. This exposes a certain inaccuracy in our analytic model that stems from the underlying treatment of the log-amplitude fluctuations' impact on the coupling efficiency. The latter is taken into account by averaging the log-amplitude spatial structures smaller than the pupil through the derivation of $\mu_{f_{\mathrm{SMF}}^{\text {sint }}}$. In doing so, the effect of these log-amplitude fluctuations on the variations of the coupling efficiency is neglected. The fact that such an assumption has a noticeable impact in the LEO rather than in the GEO scenario is not surprising, since neglecting log-amplitude fluctuations in the LEO case is fundamentally a worse approximation owing to less aperture averaging (smaller pupil diameter) combined with more challenging turbulence conditions (lower elevation). In spite of the discrepancies described above, the value of the second time derivative in the vicinity of zero predicted by the analytic model is quite close to both end-to-end and Monte Carlo results (less than 5\% of difference). This yields analytic predictions of the evolution of the AFD and AIFD with respect to the fading threshold, presented, respectively, in Figs. 10(a) and 10(b), that are in good agreement with the simulated fading durations. The AFD and AIFD corresponding to the mean level fading threshold $(-3.2 \mathrm{~dB})$ are about $2 \mathrm{~ms}$. As with the GEO downlink, the end-to-end results reveal a departure from the analytic calculation as the threshold get closer to extreme values due to lower numbers of simulated fading events in these regions.

Figures 11(a) and 11(b) show, respectively, the end-to-end FDD and IFDD for the average coupled flux threshold. The analytic geometric law still gives fairly close results for average to long fading durations. For the shorter durations, the analytic distribution is less precise, as it does not capture a clear inflection in the empirical distribution and slightly crosses the 95\% confidence bands. Such a bump in the exceedance probability of very short fade duration events means that the latter are statistically underrepresented in the end-to-end results and therefore, to the contrary, are incorrectly overrepresented by the geometric law. Although still allowing for predictions in terms of orders of magnitude, the precise origin of such a feature should be investigated further, as it may, in this scenario, question the accuracy of modeling options based on such an underlying Markovian assumption. 


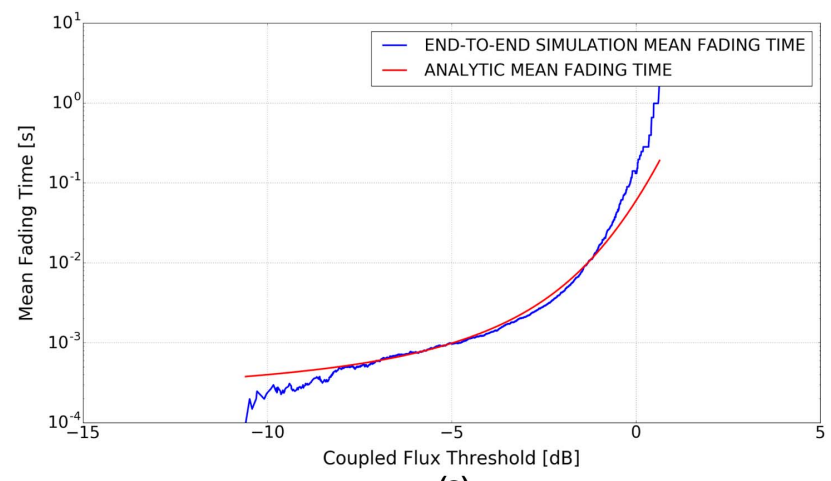

(a)

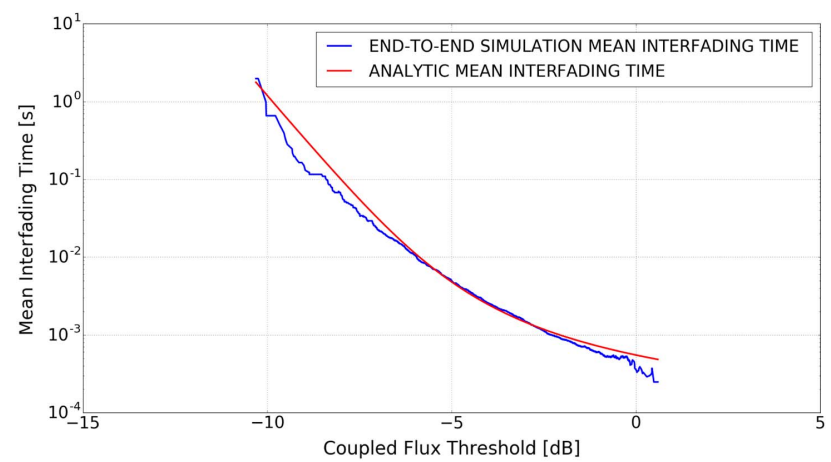

(b)

Fig. 10. Comparison of the evolution against the fading threshold of analytic and end-to-end (a) average fade duration and (b) average interfade duration for the LEO scenario.

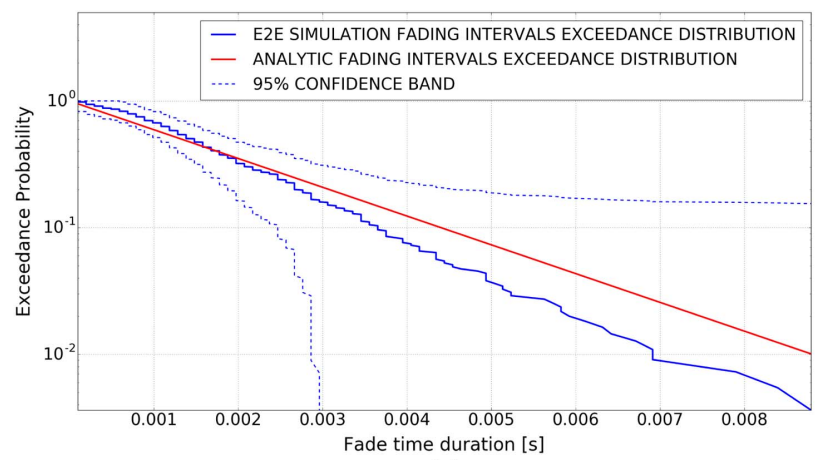

(a)

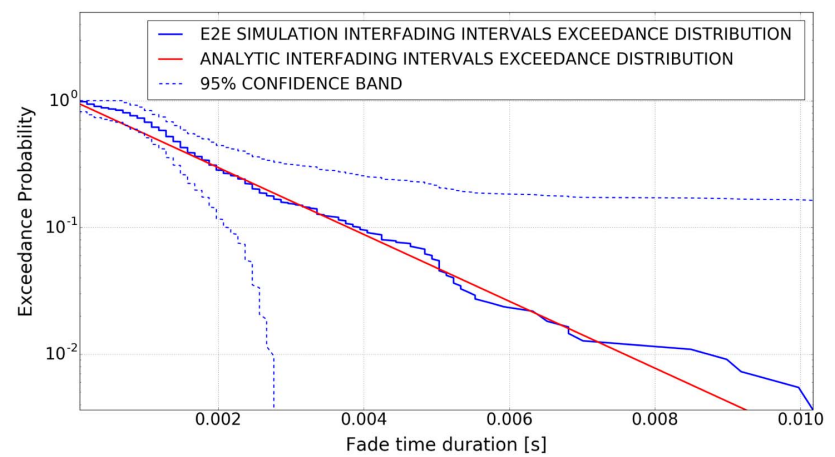

(b)

Fig. 11. Comparison of the analytic and end-to-end exceedance distributions of (a) fade duration and (b) interfade duration for a fading threshold corresponding to the average coupled flux attenuation for the LEO scenario.

\section{AO PERFORMANCE IMPACT ON FADES TEMPORAL CHARACTERISTICS}

In this section, for both the formerly discussed GEO and LEO scenarios, an analysis of the influence of three distinct $\mathrm{AO}$ system performances, denoted high (HP), medium (MP), and low performance (LP), on the analytic FDD and IFDD is presented. The specifications of the $\mathrm{AO}$ systems studied are reported in Table 2. The turbulence parameters, link parameters, as well as pupil diameters are unchanged with respect to those given in Table 1. For, respectively, the GEO and LEO downlinks, Figs. 12(a) and 12(b) show the analytic exceedance probability distributions of fading durations as well as interfading durations (dashed lines) for the mean normalized coupled flux attenuation corresponding to the different $\mathrm{AO}$ systems. This normalization is adopted in order to emphasize $\mathrm{AO}$

Table 2. AO System Parameters Corresponding to the Three Performance Levels of Both GEO and LEO Scenarios

\begin{tabular}{|c|c|c|c|c|c|}
\hline & & $N_{r}$ & $f_{s}$ & $\sigma_{\text {res }}^{2}$ & $\boldsymbol{\mu}_{f_{\text {SMF }}}$ \\
\hline \multirow{3}{*}{ GEO } & HP. & 9 & $1.0 \mathrm{kHz}$ & $0.4 \mathrm{rad}^{2}$ & $-2.7 \mathrm{~dB}$ \\
\hline & MP. & 5 & $0.4 \mathrm{kHz}$ & $1.3 \mathrm{rad}^{2}$ & $-5.2 \mathrm{~dB}$ \\
\hline & LP. & 4 & $0.3 \mathrm{kHz}$ & $1.9 \mathrm{rad}^{2}$ & $-6.7 \mathrm{~dB}$ \\
\hline \multirow{3}{*}{ LEO } & HP. & 6 & $2.0 \mathrm{kHz}$ & $0.5 \mathrm{rad}^{2}$ & $-3.2 \mathrm{~dB}$ \\
\hline & MP. & 3 & $0.8 \mathrm{kHz}$ & $1.3 \mathrm{rad}^{2}$ & $-5.2 \mathrm{~dB}$ \\
\hline & LP. & 2 & $0.5 \mathrm{kHz}$ & $2.0 \mathrm{rad}^{2}$ & $-6.7 \mathrm{~dB}$ \\
\hline
\end{tabular}

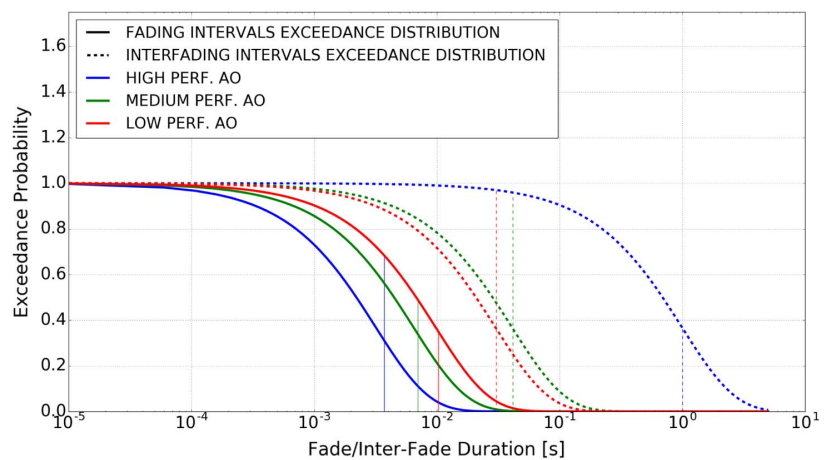

(a)

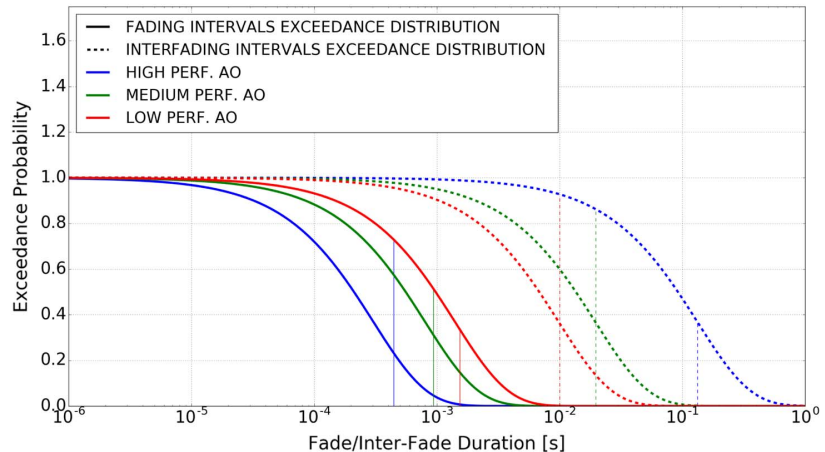

(b)

Fig. 12. Fade duration (plain lines) and interfade duration (dashed lines) analytic exceedance distributions for each AO performance level and for (a) GEO-to-ground downlink scenario and (b) LEO-toground downlink scenario. 
systems' ability to mitigate the coupled signal fluctuations only, therefore neglecting the impact of distinct $\mathrm{AO}$ systems on the average coupled flux. The AFD and AIFD corresponding to each case are directly reported on the graph by thin vertical lines. The fading thresholds chosen for computing these probability distributions are selected in order to ensure a fractional fade duration (FFD), defined as the overall proportion (in percent) of time spent below the threshold, of $0.5 \%$ for the LEO as well as GEO highest performance systems. These thresholds correspond to $-1.5 \mathrm{~dB}$ in GEO and $-5 \mathrm{~dB}$ in LEO for the highest performance systems. For the lower performance systems, thresholds of $-1.5 \mathrm{~dB}$ in GEO and $-5 \mathrm{~dB}$ in LEO yield drastically increased FFD for the less well-performing systems: $14 \%$ and $25 \%$, respectively, for the MP and LP systems in the GEO case and $4 \%$ and $14 \%$ in the LEO case. It is convenient to infer the importance of considering the impact of lowerorder residuals instead of an ideal $\mathrm{AO}$ model-characterized by its fitting error only-on the temporal characteristics of the coupled flux by considering the LP systems. Indeed, for these systems, the coupled flux fluctuations are globally dominated by the injection efficiency fluctuations. The AFDs, reported in Figs. 12(a) and 12(b), resulting from the partial AO model are roughly $10 \mathrm{~ms}$ in GEO and $1.5 \mathrm{~ms}$ in LEO. It was found that for an equivalent average level of correction and the same thresholds, neglecting the lower-order residuals yields AFDs approximately $50 \%$ smaller. The AIFDs are $10 \mathrm{~ms}$ in LEO and $30 \mathrm{~ms}$ in GEO when considering the partial $\mathrm{AO}$ correction, whereas they are, respectively, $150 \%$ and $75 \%$ larger when using an ideal AO model. These differences are not negligible when assessing data reliability mechanisms options and specifications (e.g., allowed latencies or required memory for time interleavers), especially given the very high data rates envisioned for satellite-to-ground optical communication.

On one hand, from the transmission system overall design point of view, these FFDs can be compensated by increasing the average coupled power. Not including the average loss specific to each $\mathrm{AO}$ correction, it would require for the MP and LP systems an additional 3 and $5 \mathrm{~dB}$ in GEO, 5 and $9 \mathrm{~dB}$ in $\mathrm{LEO}$, in order to reach a $0.5 \% \mathrm{FFD}$. The average loss specific to each $\mathrm{AO}$ system can be taken into account using the values of $\mu_{f_{\mathrm{SMF}}}$ reported in Table 2. For instance, in the GEO scenario, when compared to the HP correction, the MP correction requires $5.2+3-2.7=5.5 \mathrm{~dB}$ of additional mean optical power in order to guarantee a $0.5 \% \mathrm{FDD}$. On the other hand, while limiting such a recourse to increasing the average coupled power, numerical interleaving combined with FEC coding can overcome the information loss related to the decrease in $\mathrm{AO}$ correction by taking advantage of the channel correlation and adding redundancy in the transmitted data. The FFD as a metric does not capture the temporal distribution of fading events and therefore, by itself, does not allow for an assessment of interleaved and coded transmissions' reliability. For the latter, parameters such as average, maximum, and minimum fade/ interfade durations are needed as well. For the three AO performance levels presented here, Tables 3 and 4 synthesize the characteristic fade and interfade durations corresponding to $99 \%$ and $1 \%$ exceedance probabilities for, respectively, the GEO and LEO scenarios. For the GEO-to-ground link,
Table 3. Characteristic Fade and Interfade Durations for the Three Performance Levels in the GEO Scenario

\begin{tabular}{|c|c|c|c|}
\hline & & $\begin{array}{c}\text { Fading Duration } \\
{[\mathrm{ms}]}\end{array}$ & $\begin{array}{c}\text { Interfading } \\
\text { Duration [ms] }\end{array}$ \\
\hline \multirow{3}{*}{$\operatorname{Pr}\left(\tau_{\text {fade }}>\tau_{\text {fade }}^{0}\right)=.99$} & HP. & 0.032 & 10 \\
\hline & MP. & 0.065 & 0.4 \\
\hline & LP. & 0.098 & 0.3 \\
\hline \multirow{3}{*}{$\operatorname{Pr}\left(\tau_{\text {fade }}>\tau_{\text {fade }}^{0}\right)=.01$} & HP. & 15 & 4500 \\
\hline & MP. & 30 & 190 \\
\hline & LP. & 45 & 140 \\
\hline
\end{tabular}

Table 4. Characteristic Fade and Interfade Durations for the Three Performance Levels in the LEO Scenario

\begin{tabular}{|c|c|c|c|}
\hline & & $\begin{array}{c}\text { Fading } \\
\text { Duration [ms] }\end{array}$ & $\begin{array}{c}\text { Inter-Fading } \\
\text { Duration [ms] }\end{array}$ \\
\hline $\operatorname{Pr}\left(\tau_{\text {fade }}>\tau_{\text {fade }}^{0}\right)=.99$ & $\begin{array}{l}\text { HP. } \\
\text { MP. } \\
\text { LP. }\end{array}$ & $\begin{array}{l}0.003 \\
0.008 \\
0.014\end{array}$ & $\begin{array}{l}1.3 \\
0.2 \\
0.1\end{array}$ \\
\hline $\operatorname{Pr}\left(\tau_{\text {fade }}>\tau_{\text {fade }}^{0}\right)=.01$ & $\begin{array}{l}\text { HP. } \\
\text { MP. } \\
\text { LP. }\end{array}$ & $\begin{array}{l}1.4 \\
3.7 \\
6.4\end{array}$ & $\begin{array}{c}611 \\
90 \\
45\end{array}$ \\
\hline
\end{tabular}

dividing the total residual variance $\sigma_{\text {res }}^{2}$ of the HP system by a factor of 3 or 5 , respectively, increases the minimum, maximum and AFDs by a factor of 2 or 3 . This may seem a notably small improvement in light of the specifications required by the HP system. However, the gain with respect to the interfading durations is remarkably significant, as the HP system outperforms by several orders of magnitude the other $\mathrm{AO}$ corrections, hence, not only making fading events shorter to some extent, but drastically spreading them further apart. Regarding the LEO downlink, reducing the total residual phase variance of the HP system in roughly the same proportions as for the GEO case leads to similar observations in terms of fading durations and their distribution. The impact of $\mathrm{AO}$ performance on interfading durations is again striking, since that downgrading from the HP to the MP and LP systems yields, respectively, maximum, minimum, and AIFDs roughly 10 times smaller.

Finally, in each scenario, the MP and LP AO systems present close performances in terms of temporal characteristics that are relevant for the optimization of numerical mitigation techniques. The HP AO systems, on the contrary, seem to mitigate fading events more acutely at the cost of only roughly twice the control-loop frequency and 4 times the number of actuators of the medium correction. These results highlight the importance, for AO-corrected laser links, of not solely considering average $\mathrm{AO}$ performance parameters such as the residual variance or average attenuation, originally used in the context of astronomy or imagery applications. Furthermore, analyses such as the one exposed in this section emphasize the interest of the presented analytical model, which provides a relevant order of magnitudes, if not accurate results, while avoiding timeconsuming and complex simulations. 


\section{CONCLUSION}

An analytical model describing the temporal variations of the coupled flux into a SMF for satellite-to-ground laser links partially corrected by $\mathrm{AO}$ has been presented. The modeling of the $\mathrm{AO}$ correction is representative of a realistic error budgeting that includes fitting, aliasing, as well as temporal errors of the system. Taking advantage of the decomposition of the residual phase over an orthonormal basis of polynomials, an approximation of the coupling efficiency of partially corrected wavefronts into an SMF has been derived. Furthermore, in order to take into account scintillation effects in addition to the residual phase fluctuations on the instantaneous coupled flux, an approximation of the latter has been derived by considering the aperture-averaged log-amplitude spatial structures smaller than the pupil. Analytical expressions of its PDF and $\mathrm{CDF}$ as well as an estimation of its temporal autocovariance have been provided. These results were exploited in order to obtain relevant temporal parameters such as the average fading/ interfading duration and fading/interfading durations distribution. All of these analytical developments have been confronted with end-to-end wave-optics simulations in the case of GEO as well as LEO-to-ground link scenarios. In the case of the former, this confrontation validated the underlying physical hypotheses made. In the LEO case, due to the more challenging conditions of this scenario with respect to the impact of scintillation on the coupled flux (smaller aperture and lower elevation), our analytical model seems to be statistically less accurate, providing, nonetheless, a reasonable order of magnitudes. Eventually, a case study illustrating the relevance of such a model in evaluating analytically the performance of three distinct $\mathrm{AO}$ systems while emphasizing characteristic fading and interfading durations has been presented. The latter constitutes intermediate yet essential parameters for the computation of coded-transmission performance metrics over correlated channels, such as, for instance, the packet error rate after imperfect interleaving. Additionally, in the framework of joint-optimization studies of $\mathrm{AO}$ and numerical mitigation techniques, these characteristic durations should always be investigated, since it has been shown that considering typical AO correction estimators such as the residual phase variance might not entirely suffice to infer the influence of $\mathrm{AO}$ on telecom performance. More precisely, it has been shown that a significant gain in terms of interfading durations is expected from the use of $\mathrm{HP} \mathrm{AO}$ systems in addition to a much more favorable average power penalty.

\section{APPENDIX A: MODAL DECOMPOSITION OF THE COUPLED TURBULENT PHASE ONTO AN ORTHONORMAL BASIS}

The efficient yet simple method derived in $[14,15]$ for computing a conversion matrix linking the decomposition coefficients of the Zernike polynomials to the coefficients of a new basis of orthonormal polynomials is summarized hereafter. Let us first write the complete set of Zernike polynomials as $\left\{Z_{i}\right\}$ over the intensity domain $\Sigma$ comprising a circular pupil and the backpropagated Gaussian SMF on the pupil as well. Because of the completeness of $\left\{Z_{i}\right\}$, one can represent any other set of polynomials as a linear combination of $\left\{Z_{i}\right\}$, including a set $\left\{F_{i}\right\}$ that is indeed orthonormal over $\Sigma$. These two sets are related to each other according to

$$
F_{i}=\sum_{j=1}^{N} M_{i j} Z_{j}
$$

where $\mathbf{M}$ is a conversion matrix and $N$ is the number of polynomials arbitrarily determined in order to represent a given aberration. One can always write

$$
\left\langle F_{i} \mid Z_{k}\right\rangle_{W_{0}}=\sum_{j=1}^{N} M_{i j}\left\langle Z_{j} \mid Z_{k}\right\rangle_{W_{0}}
$$

or

$$
\left\langle Z_{k} \mid F_{i}\right\rangle_{W_{0}}=\sum_{j=1}^{N}\left[M_{i j}\left\langle Z_{j} \mid Z_{k}\right\rangle_{W_{0}}\right]^{T}=\sum_{j=1}^{N}\left\langle Z_{k} \mid Z_{j}\right\rangle_{W_{0}}\left[M_{i j}\right]^{T}
$$

written in matrix form as

$$
\mathbf{C}^{Z F}=\mathbf{C}^{Z Z} \mathbf{M}^{T},
$$

where $\mathbf{C}^{Z F}$ is an $N \times N$ matrix of the inner products between the first $N$ polynomials of $Z_{k}$ and the first $N$ polynomials of $F_{i}$, and $\mathbf{C}^{Z Z}$ is an $N \times N$ matrix of the inner products between the first $N$ polynomials of $Z_{k}$ with themselves. Similarly, one can write

$$
\left\langle F_{i} \mid F_{k}\right\rangle_{W_{0}}=\sum_{j=1}^{N} M_{i j}\left\langle Z_{j} \mid Z_{k}\right\rangle_{W_{0}}=\delta_{i k},
$$

where $\delta_{i k}$ is the Kronecker symbol, and which can be written in matrix form as well:

$$
\mathbf{M C}^{Z F}=\mathbf{1}
$$

Substituting Eq. (A4) in Eq. (A6) yields

$$
\mathbf{M C}^{Z Z} \mathbf{M}^{T}=\mathbf{1} \text {. }
$$

Now, let

$$
\mathbf{M}=\left(\mathbf{Q}^{T}\right)^{-1} .
$$

Then Eq. (A7) can be written as

$$
\mathbf{Q}^{T} \mathbf{Q}=\mathbf{C}^{Z Z} \text {. }
$$

The inner product matrix $\mathbf{C}^{Z Z}$ is symmetric positive definite. Therefore Eq. (A9) can be solved for $\mathbf{Q}$ uniquely with a simple Cholesky decomposition, and eventually the conversion matrix $\mathbf{M}$ can be obtained from Eq. (A8). The inverse of the transpose of this conversion matrix, $\left(\mathbf{M}^{T}\right)^{-1}$, is required to express the orthonormal coefficients in terms of the original Zernike coefficients [see Eq. (20)]. This matrix (the absolute values of its elements) is represented in Fig. 13 for the first 50 modes (piston included). One can see that it is nearly diagonal, but terms with the same azimuthal frequency such as tip-tilt and comas or piston, defocus, and spherical aberration are not completely null and hence induce some correlation among the orthonormal coefficients. The latter is nevertheless relatively weak and can be neglected in a first approximation. 


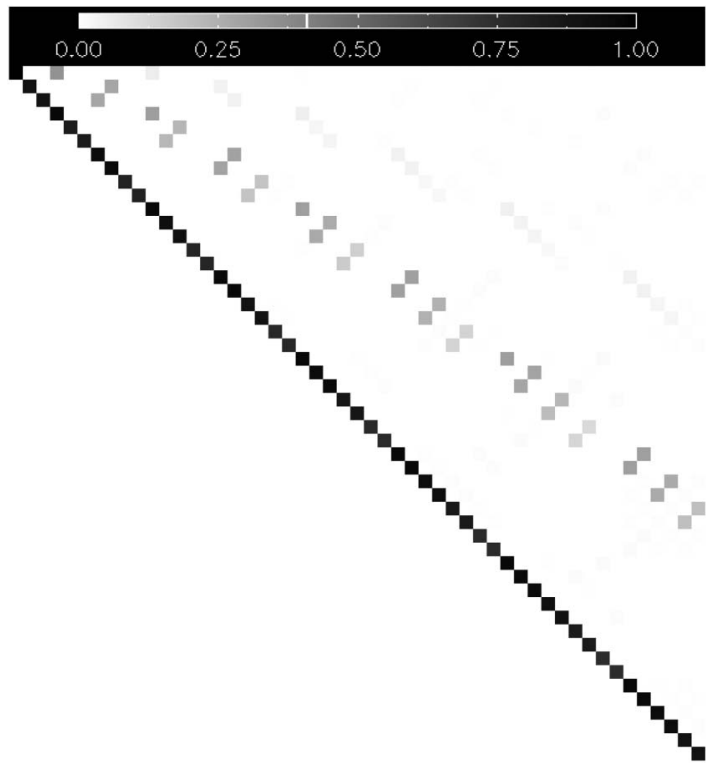

Fig. 13. Absolute values of the inverse of the transpose of the conversion matrix, $\left|\left(\mathbf{M}^{T}\right)^{-1}\right|$.

\section{APPENDIX B: AVERAGE COUPLED FLUX WITH A FULL AO CORRECTION}

Adapting the results related to the average of the long-exposure images' modulation transfer function presented in [20], an expression for the average coupled flux into an SMF while neglecting phase aberrations of the turbulent wavefront is given by

$\mu_{f_{\text {SMF }}^{\text {sint }}}=\frac{A_{0}^{2}}{\rho_{0}} \exp \left(-C_{\chi}(0)\right) \iint W_{0}(\mathbf{r}) W_{0}(\mathbf{r}+\mathbf{s}) \exp \left(C_{\chi}(\mathbf{s})\right) \mathrm{d}^{2} \mathbf{r d}^{2} \mathbf{s}$.

This equation can always be written as

$$
\mu_{f_{\mathrm{SMF}}^{\text {sint }}}=\frac{A_{0}^{2}}{\rho_{0}} \exp \left(-C_{\chi}(0)\right) \int \exp \left(C_{\chi}(\mathbf{s})\right)\left(W_{0} * W_{0}\right)(\mathbf{s}) \mathrm{d}^{2} \mathbf{s},
$$

where the symbol $*$ denotes convolution. The term $\left(W_{0} *\right.$ $\left.W_{0}\right)($ s) corresponds to the autocorrelation of the apodized pupil (i.e., weighted by the Gaussian mode of the SMF), which is in fact the transfer function of the instrument (i.e., telescope and SMF). Approximating $\exp \left(C_{\chi}(\mathbf{s})\right)$ by its first two series terms yields

$$
\begin{aligned}
\mu_{f_{\text {SMF }}^{\text {Sint }}} \simeq & \frac{A_{0}^{2}}{\rho_{0}} \exp \left(-C_{\chi}(0)\right)\left(\int\left(W_{0} * W_{0}\right)(\mathbf{s}) \mathrm{d}^{2} \mathbf{s}\right. \\
& \left.+\int C_{\chi}(\mathbf{s})\left(W_{0} * W_{0}\right)(\mathbf{s}) \mathrm{d}^{2} \mathbf{s}\right) .
\end{aligned}
$$

This approximation tends to be less accurate, as the logamplitude fluctuation regime is stronger. Using the generalized Parseval theorem, and remembering that $\left\langle A_{0} \mid A_{0}\right\rangle_{P}=1$, yields

$$
\begin{aligned}
\mu_{f_{\text {SMF }}^{\text {Sint }}} \simeq & \exp \left(-C_{\chi}(0)\right) \\
& \times\left(1+\frac{\int \mathcal{F}\left[C_{\chi}(\mathbf{r})\right](\boldsymbol{\omega})\left[\mathcal{F}\left[W_{0}(\mathbf{r})\right](\boldsymbol{\omega})\right]^{2} \mathrm{~d}^{2} \boldsymbol{\omega}}{\rho_{0} \times\langle 1 \mid 1\rangle_{P}}\right),
\end{aligned}
$$

where the symbol $\mathcal{F}$ denotes the Fourier transform. Note that the term $\mathcal{F}\left[C_{\chi}(\mathbf{r})\right](\boldsymbol{\omega})\left[\mathcal{F}\left[W_{0}(\mathbf{r})\right](\boldsymbol{\omega})\right]^{2} d^{2} \boldsymbol{\omega}$ corresponds to the spectral power density of the log-amplitude fluctuations in the pupil filtered by the instrument. It represents the influence of the spatial correlation of the speckles in the pupil on the coupled flux attenuation.

Funding. Centre National d'Etudes Spatiales (CNES); Thales Group (Thales) Alenia Space; Airbus Defense and Space.

Acknowledgment. This work was conducted in the framework of a PhD thesis supervised by ISAE-Supaéro and ONERA, and co-funded by CNES, Airbus Defense and Space, and Thales Alenia Space.

\section{REFERENCES}

1. J. Ma, L. Ma, Q. Yang, and Q. Ran, "Statistical model of the efficiency for spatial light coupling into a single-mode fiber in the presence of atmospheric turbulence," Appl. Opt. 54, 9287-9293 (2015).

2. A. Belmonte and J. M. Kahn, "Performance of synchronous optical receivers using atmospheric compensation techniques," Opt. Express 16, 14151-14162 (2008).

3. J. W. Goodman, Speckle Phenomena in Optics: Theory and Applications (Ben Roberts, 2007).

4. G. A. Tyler, "Assessment of the statistics of the Strehl ratio: predictions of central limit theorem analysis," J. Opt. Soc. Am. A 23, 2834-2844 (2006).

5. C. Ruilier and F. Cassaing, "Coupling of large telescopes and singlemode waveguides: application to stellar interferometry," J. Opt. Soc. Am. 18, 143-149 (2001).

6. A. Maréchal, "Étude des effets combinés de la diffraction et des aberrations géometriques sur l'image d'un point lumineux," Rev. Opt. Theor. Instrum. 26, 257-277 (1947).

7. J. Huang, C. Liu, K. Deng, Z. Yao, H. Xian, and X. Li, "Probability of the residual wavefront variance of an adaptive optics system and its application," Opt. Express 24, 2818-2829 (2016).

8. S. Gladysz, J. C. Christou, L. W. Bradford, and L. C. Roberts, Jr., "Temporal variability and statistics of the Strehl ratio in adaptive-optics images," Publ. Astron. Soc. Pac. 120, 1132-1143 (2008).

9. R. J. Noll, "Zernike polynomials and atmospheric turbulence," J. Opt. Soc. Am. 66, 207-211 (1976).

10. C. Ruilier, "A study of degraded light coupling into single-mode fibers," Proc. SPIE 3350, 319-329 (1998).

11. S. Shaklan and F. Roddier, "Coupling starlight into single-mode fiber optics," Appl. Opt. 27, 2334-2338 (1988).

12. S. Meimon, F. Cassaing, and G. Prévôt, "Experimental study of distorted beams coupling in a single mode waveguide," J. Opt. 15, 035707 (2013).

13. V. N. Mahajan, ed., Optical Imaging and Aberrations. Part III: Wavefront Analysis (SPIE, 2013).

14. G. Dai and V. N. Mahajan, "Nonrecursive determination of orthonormal polynomials with matrix formulation," Opt. Lett. 32, 74-76 (2007).

15. G. Dai and V. N. Mahajan, "Orthonormal polynomials in wavefront analysis: error analysis," Appl. Opt. 47, 3433-3445 (2008).

16. N. Vedrenne, J.-M. Conan, C. Petit, and V. Michau, "Adaptive optics for high data rate satellite to ground laser link," Proc. SPIE 9739 97390E (2016).

17. N. A. Roddier, "Atmospheric wavefront simulation using Zernike polynomials," Opt. Eng. 29, 1174-1181 (1990). 
18. J.-M. Conan, G. Rousset, and P.-Y. Madec, "Wave-front temporal spectra in high-resolution imaging through turbulence," J. Opt. Soc. Am. A 12, 1559-1570 (1995).

19. S. Cha, "Comprehensive survey on distance/similarity measures between probability density functions," Int. J. Math. Models Methods Appl. Sci. 1, 300-307 (2007).

20. D. L. Fried, "Optical resolution through a randomly inhomogeneous medium for very long and very short exposures," J. Opt. Soc. Am. 56, 1372-1379 (1966).

21. V. Tatarski, Wave Propagation in a Turbulent Medium (Dover, 1961).

22. J. W. Strohbehn, Modern Theories in the Propagation of Optical Waves in a Turbulent Medium (Springer, 1978), pp. 45-106.

23. B. Epple, "Simplified channel model for simulation of free-space optical communications," J. Opt. Commun. Netw. 2, 293-304 (2010).

24. C. Robert, J.-M. Conan, V. Michau, J.-B. Renard, C. Robert, and F. Dalaudier, "Retrieving parameters of the anisotropic refractive index fluctuations spectrum in the stratosphere from balloon-borne observations of stellar scintillation," J. Opt. Soc. Am. A 25, 379-393 (2008).

25. N. Perlot, "Turbulence-induced fading probability in coherent optical communication through the atmosphere," Appl. Opt. 46, 7218-7226 (2007)

26. F. Roddier, "V the effects of atmospheric turbulence in optical astronomy," Prog. Opt. 19, 281-376 (1981).

27. J. Gil-Pelaez, "Note on the inversion theorem," Biometrika 38, 481-482 (1951).

28. G. Efthymoglou and V. Aalo, "Performance of RAKE receivers in Nakagami fading channel with arbitrary fading parameters," Electron. Lett. 31, 1610-1612 (1995).

29. I. S. Ansari, F. Yilmaz, M. S. Alouini, and O. Kucur, "On the sum of gamma random variates with application to the performance of maximal ratio combining over Nakagami-m fading channels," in 13th International Workshop on Signal Processing Advances in Wireless Communications (SPAWC) (2012), pp. 394-398.

30. V. A. Aalo, T. Piboongungon, and G. P. Efthymoglou, "Another look at the performance of MRC schemes in Nakagami-m fading channels with arbitrary parameters," IEEE Trans. Commun. 53, 2002-2005 (2005).

31. L. Canuet, N. Vedrenne, J.-M. Conan, G. Artaud, A. Rissons, and J. Lacan, "Evaluation of communication performance for adaptive optics corrected geo-to-ground laser links," Proc. SPIE 10562, 1056248 (2016).

32. L. Isserlis, "On certain probable errors and correlation coefficients of multiple frequency distributions with skew regression," Biometrika 11, 185-190 (1916).

33. L. Isserlis, "On a formula for the product-moment coefficient of any order of a normal frequency distribution in any number of variables," Biometrika 12, 134-139 (1918).

34. M. R. Whiteley, M. C. Roggemann, and B. M. Welsh, "Temporal properties of the Zernike expansion coefficients of turbulence-induced phase aberrations for aperture and source motion," J. Opt. Soc. Am. A 15, 993-1005 (1998).

35. M. S. Bartlett, "On the theoretical specification and sampling properties of autocorrelated time-series," Suppl. J. R. Stat. Soc. 8, 27-41 (1946).

36. W. E. Wecker, "A note on the time series which is the product of two stationary time series," Stochastic Process. Appl. 8, 153-157 (1978).
37. H. T. Yura and S. G. Hanson, "Mean level signal crossing rate for an arbitrary stochastic process," J. Opt. Soc. Am. A 27, 797-807 (2010).

38. J. S. Bendat and A. G. Piersol, Stationary Random Processes (Wiley, 2012), pp. 109-171.

39. J. P. Adoul, "Error intervals and cluster density in channel modeling (corresp.)," IEEE Trans. Inf. Theory 20, 125-129 (1974).

40. L. Wilhelmsson and L. B. Milstein, "On the effect of imperfect interleaving for the Gilbert-Elliott channel," IEEE Trans. Commun. 47, 681-688 (1999).

41. G. Liva, B. Matuz, Z. Katona, E. Paolini, and M. Chiani, "On construction of moderate-length LDPC codes over correlated erasure channels," in IEEE International Conference on Communications (2009), pp. 1-5.

42. H. Henniger, "Transmission performance analysis of free-space optical communications using Gilbert-erasure channel," IEEE Trans. Commun. 60, 55-61 (2012).

43. A. Puryear, R. Jin, E. Lee, and V. W. S. Chan, "Experimental analysis of the time dynamics of coherent communication through turbulence: Markovianity and channel prediction," in International Conference on Space Optical Systems and Applications (ICSOS) (2011), pp. 28-37.

44. E. J. Lee and V. W. S. Chan, "Performance of the transport layer protocol for diversity communication over the clear turbulent atmospheric optical channel," in IEEE International Conference on Communications (ICC) (2005), vol. 1, pp. 333-339.

45. N. Vedrenne, M. T. Velluet, C. Petit, V. Michau, J. Chabe, A. Ziad, D. H. Phung, N. Maurice, E. Samain, G. Artaud, J. L. Issler, M. Toyoshima, M. Akioka, D. Kolev, Y. Munemasa, H. Takenaka, and N. Iwakiri, "First results of wavefront sensing on SOTA," in IEEE International Conference on Space Optical Systems and Applications (ICSOS) (2015), pp. 1-8.

46. M. Toyoshima, H. Takenaka, Y. Shoji, and Y. Takayama, "Frequency characteristics of atmospheric turbulence in space-to-ground laser links," Proc. SPIE 7685, 76850G (2010).

47. V. V. Voitsekhovich, V. G. Orlov, and L. J. Sanchez, "Influence of scintillations on the performance of adaptive astronomical systems with Hartmann-like wavefront sensors," Astron. Astrophys. 368, 1133-1136 (2001).

48. F. Fidler, M. Knapek, J. Horwath, and W. R. Leeb, "Optical communications for high-altitude platforms," J. Sel. Top. Quantum Electron. 16, 1058-1070 (2010).

49. M. A. Stephens, "Use of the Kolmogorov-Smirnov, Cramer-Von Mises and related statistics without extensive tables," J. R. Stat. Soc. Ser. B 32, 115-122 (1970)

50. C. R. Jerkins and J. V. Wall, Practical Statistics for Astronomers, Vol. 3 of Cambridge Observing Handbooks for Research Astronomers, 2nd ed. (Cambridge University, 2012).

51. M. Priestley, Spectral Analysis and Time Series, Probability and Mathematical Statistics (Academic, 1989).

52. P. M. T. Broersen, "Estimation of the accuracy of mean and variance of correlated data," in IEEE Instrumentation and Measurement Technology Conference (IMTC/98). Where Instrumentation Is Going (1998), vol. 1, pp. 36-41.

53. J. R. Yee and E. J. Weldon, "Evaluation of the performance of errorcorrecting codes on a Gilbert channel," IEEE Trans. Commun. 43, 2316-2323 (1995). 